DESY-12-161

\title{
NLO Corrections to the kernel of the BKP-equations
}

\author{
J. Bartels ${ }^{a}$, V.S.Fadin ${ }^{b}$, L.N.Lipatov ${ }^{a, c}$, G.P.Vacca $^{d}$ \\ ${ }^{a}$ II. Institut für Theoretische Physik, Universität Hamburg, Luruper Chaussee 149, \\ D-22761 Hamburg, Germany \\ ${ }^{b}$ Budker Institute of Nuclear Physics, 630090 Novosibirsk, Russia, and Novosibirsk State \\ University, 630090 Novosibirsk, Russia \\ ${ }^{c}$ Petersburg Nuclear Physics Institute, Gatchina 188300, St.Peterburg, Russia \\ ${ }^{d}$ INFN Sezione di Bologna, via Irnerio 46, I-40126 Bologna, Italy
}

\begin{abstract}
:
We present results for the NLO kernel of the BKP equations for composite states of three reggeized gluons in the Odderon channel, both in QCD and in N=4 SYM. The NLO kernel consists of the NLO BFKL kernel in the color octet representation and the connected $3 \rightarrow 3$ kernel, computed in the tree approximation.
\end{abstract}

\section{Introduction}

The BFKL equation [1] describing the bound states of two reggeized gluons represents one of the fundamental equations in QCD. The kernel has been computed in NLO accuracy both for the colour singlet $t$-channel state in the forward direction (i.e. at $t=0$ ) [2, 3], and for arbitrary $t$ and other possible colour configurations in the $t$-channel [4, 5, 6]. In the forward case the solutions of the equation have been investigated intensively. Most attention has been given to the color singlet channel, but recently, motivated by the AdS/CFT correspondence, also the color octet channel has been investigated [7].

The generalization of the BFKL equation to bound states consisting of three and more reggeized gluons, the BKP equation [8], has attracted strong interest, because, in the large- $N_{c}$ limit and in leading order of $\alpha_{3}$, it has been found to be integrable [9, 10]. Integrability plays a vital role in analyzing the conjectured correspondence between $N=4$ SYM and string theory. A particular case of the BKP equations, the C-odd three gluon system, is also of some practical interest: in leading order it predicts the existence of a (perturbative) Odderon with intercept one [11. The accuracy of the BKP equations, so far, has been limited to the leading logarithmic approximation (LO). Clearly it is important to find out whether integrability holds beyond the leading order. Also, the Odderon intercept at NLO is of interest: if it would remain at unity, it would hint at some deeper property of the Odderon system. 
It is the purpose of this paper to compute the kernel for the BKP equations in the Odderon channel in the NLO approximation. In this approximation

$$
K=K(2 \rightarrow 2)+K(3 \rightarrow 3)
$$

where $K(2 \rightarrow 2)$ is the sum over NLO $2 \rightarrow 2$ kernels, and $K(3 \rightarrow 3)$ is the connected $3 \rightarrow 3$ kernel computed in the Born approximation. These building blocks of the NLO kernel are illustrated in Fig.1.
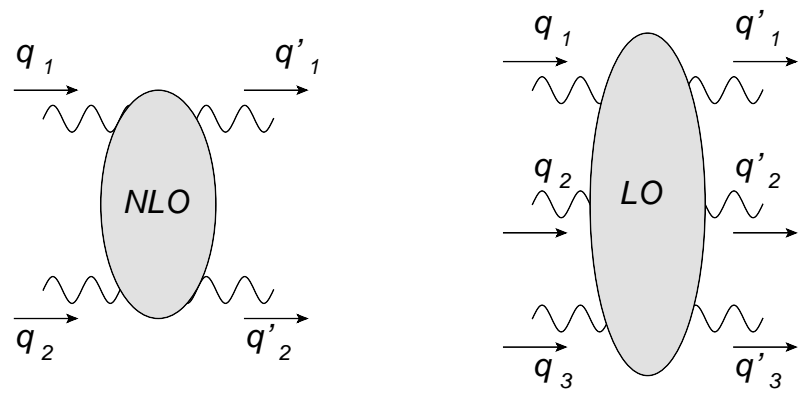

Fig.1: The building blocks of the NLO contributions to the BKP equations; $\vec{q}_{i}$ and $\vec{q}_{i}^{\prime}$ are the reggeized gluon momenta transverse to the momenta of colliding particles.

Since in the C-odd three gluon system any pair of two gluons forms symmetric colour octet subsystems, the NLO $2 \rightarrow 2$ kernel is related to the NLO BFKL kernel in the symmetric color octet representation, though it does not coincide with it. We remind that for any colour representation the BFKL kernel can be written as the sum of a "virtual" part containing gluon Regge trajectories and a "real" contribution, which results from the $s$ channel intermediate states [1, 12]. In contrast to the BFKL equation which is written for two interacting reggeized gluons and, therefore, contains two trajectories and the real contribution, the BKP equation for the Odderon describes the interaction of three reggeized gluons. Thus it contains three trajectories and three real parts corresponding to the $s$-channel exchanges between various pairs of the reggeized gluons. Writing the $2 \rightarrow 2$ kernel as the sum of the coupled kernels

$$
K(2 \rightarrow 2)=K_{12}+K_{23}+K_{13},
$$

we obtain that the kernels $K_{i j}$ have the same real parts as the BFKL kernels for the reggeized gluons $i$ and $j$ in the symmetric colour octet representation, but their virtual parts are two times smaller. Note that just this property provides the infrared stability of the kernels $K_{i j}$ as a consequence of the fact that the Odderon is a colorless state.

The $3 \rightarrow 3$ kernel is new: in the Born approximation it contains only gluons and, hence, it is the same for QCD and for supersymmetric Yang-Mills theories. The calculation starts from the effective action [13, 14] and requires the use of Ward identities of amplitudes of reggeized gluons. These Ward identities have been derived recently [15], and they play an essential role in the present paper.

This paper consists of two parts. Section 2 contains the NLO BFKL kernel in the octet representation, and in section 3 we present the new $3 \rightarrow 3$ vertex. Some details of our calculations are collected in an Appendix. 


\section{The $2 \rightarrow 2$ Kernel}

The $2 \rightarrow 2$ kernel, $K(2 \rightarrow 2)$, for the three-gluon system is given by the sum (2). Following the discussion after (2) and using the representation of the NLO BFKL kernel given in [12] we have, for the color octet representations,

$$
K_{12}^{f, d}=\frac{1}{2} \delta\left(\vec{q}_{1}-\vec{q}_{1}^{\prime}\right) \vec{q}_{1}^{2} \vec{q}_{2}^{2}\left(\omega\left(-\vec{q}_{1}^{2}\right)+\omega\left(-\vec{q}_{2}^{2}\right)\right)+K_{r}^{f, d}\left(\vec{q}_{1}, \vec{q}_{1}^{\prime} ; \vec{q}\right)
$$

where $\vec{q}=\vec{q}_{1}+\vec{q}_{2}=\vec{q}_{1}^{\prime}+\vec{q}_{2}^{\prime}, \omega\left(-\vec{q}_{i}^{2}\right)$ is the reggeized gluon trajectory, and $K_{r}^{f, d}\left(\vec{q}_{1}, \vec{q}_{1}^{\prime} ; \vec{q}\right)$ denotes the real part of the BFKL kernel in the two different octet representation. The superscripts $f$ and $d$ refer to the antisymmetric ( $f$-coupling) and symmetric ( $d$ coupling) adjoint representations, resp. For the Odderon we are interested in the symmetric octet representation, $K_{12}^{d}$, but as it will become clear in the following, it will be easier to start with the antisymmetric case. It was already mentioned that the kernels $K_{12}$ are infrared stable, though taken separately the trajectory [16] and the real parts [4], 5] are strongly infrared divergent. It turns out feasible [17] to perform the cancelation of the divergencies explicitly and to write the kernel in the physical two-dimensional transverse momentum space. To do it one has to use the integral representation for the trajectory. For QCD such a representation is given in [18]. Keeping in mind that we are interested also in supersymmetric Yang-Mills theories, we generalize QCD to more general theories with $n_{f}$ spin-one-half fermions and $n_{s}$ scalars in arbitrary representations of the colour group. For such theories, using results of [19], we obtain in $D=4+2 \epsilon$ space time dimensions

$$
\omega\left(-\vec{q}_{i}^{2}\right)=\frac{-\bar{g}^{2} \vec{q}_{i}^{2}}{\pi^{1+\epsilon} \Gamma(1-\epsilon)} \int \frac{d^{2+2 \epsilon} k}{\vec{k}^{2}\left(\vec{k}-\vec{q}_{i}\right)^{2}}\left(1+\bar{g}^{2}\left[f(\vec{k}, 0)+f\left(0, \vec{k}-\vec{q}_{i}\right)-f\left(\vec{k}, \vec{k}-\vec{q}_{i}\right)\right]\right)
$$

where

$$
\bar{g}^{2}=\frac{g^{2} N_{c} \Gamma(1-\epsilon)}{(4 \pi)^{2+\epsilon}} .
$$

$\Gamma(x)$ is the Euler gamma-function, $g$ is the bare coupling, and the function $f$ can be written as

$$
f\left(\vec{k}_{1}, \vec{k}_{2}\right)=\frac{\vec{k}_{12}^{2}}{\pi^{1+\epsilon} \Gamma(1-\epsilon)} \int \frac{d^{2+2 \epsilon} l}{\left(\vec{k}_{1}-\vec{l}\right)^{2}\left(\vec{k}_{2}-\vec{l}\right)^{2}}\left(\ln \left(\frac{\vec{k}_{12}^{2}}{\vec{l}^{2}}\right)+a\right)
$$

with $\vec{k}_{12}=\vec{k}_{1}-\vec{k}_{2}$. The constant $a$ has the form:

$$
\begin{gathered}
a=-2 \psi(1+2 \epsilon)-\psi(1-\epsilon)+2 \psi(\epsilon)+\psi(1)+\frac{1}{(1+2 \epsilon)}\left(\frac{1}{\epsilon}+\frac{1+\epsilon}{2(3+2 \epsilon)}\right) \\
+\frac{a_{f}}{N_{c}} \frac{1+\epsilon}{(1+2 \epsilon)(3+2 \epsilon)}+\frac{a_{s}}{N_{c}} \frac{1}{4(1+2 \epsilon)(3+2 \epsilon)} .
\end{gathered}
$$

Here $\psi(x)=\Gamma^{\prime}(x) / \Gamma(x), a_{f}=2 \kappa_{f} n_{f} T_{f}, \quad a_{s}=2 \kappa_{s} n_{s} T_{s}, T_{f}$ and $T_{s}$ are defined by the relations

$$
\operatorname{Tr}\left(T_{f}^{a} T_{f}^{b}\right)=T_{f} \delta^{a b}, \quad \operatorname{Tr}\left(T_{s}^{a} T_{s}^{b}\right)=T_{s} \delta^{a b},
$$


where $T_{f}^{a}$ and $T_{s}^{a}$ are the colour group generators for fermions and scalars, respectively, and $\kappa_{f}\left(\kappa_{s}\right)$ is equal to $1 / 2$ for Majorana fermions (neutral scalars) in self-conjugated representations and 1 otherwise. In the case of $n_{M}$ Majorana fermions and $n_{s}$ squarks in the adjoint representation we put $a_{f} \rightarrow n_{M} N_{c}, a_{s} \rightarrow n_{s} N_{c}$. For $N$-extended SYM $n_{M}=N, \quad n_{s}=2(N-1)$. In the $\overline{\mathrm{MS}}$ scheme the bare coupling is connected with the renormalized coupling, $g_{\mu}$, through the relation

$$
g=g_{\mu} \mu^{-\epsilon}\left[1+\bar{g}_{\mu}^{2} \frac{\beta_{0}}{2 N_{c} \epsilon}\right], \quad \bar{g}_{\mu}^{2}=\frac{g_{\mu}^{2} N_{c} \Gamma(1-\epsilon)}{(4 \pi)^{2+\epsilon}}, \quad \beta_{0} N_{c}=\frac{11}{3} N_{c}-\frac{2}{3} a_{f}-\frac{1}{6} a_{s} .
$$

As it is known, dimensional regularization violates supersymmetry, and in supersymmetric theories a modification of dimensional regularization is used which is called dimensional reduction. With our accuracy the use of the dimensional reduction (instead of the dimensional regularization) is equivalent to the finite charge remormalization

$$
\alpha_{s}(\mu) \rightarrow \alpha_{s}(\mu)\left(1-\frac{\alpha_{s}(\mu) N_{c}}{12 \pi}\right) .
$$

The expressions (6) and (7) are exact in $\epsilon$.

The real part of the NLO BFKL kernel, $K_{r}^{f, d}$, contains gluon, fermion, and scalar contributions. Contrary to the gluon contribution [5] to $K_{r}$ for which the symmetric and antisymmetric color octet representations coincide, fermions [4] and scalars [19] give different contributions for these representations. For the fermionic and scalar contributions to $K_{r}^{d}$ one finds

$$
K_{f, s}^{d}=K_{f, s}^{f}+K_{f, s}^{a}
$$

such that

$$
K_{r}^{d}=K_{r}^{f}+K_{s}^{a}+K_{f}^{a} .
$$

Here $K_{f, s}^{a}$ are the so-called abelian parts. They are given by closed fermion and scalar loops, and up to coefficients $\left(g_{\mu}^{4} / e^{4}\right) b_{f, s}$,

$$
b_{f, s}=\kappa_{f, s} \frac{N_{c} n_{f, s}}{2\left(N_{c}^{2}-4\right)\left(N_{c}^{2}-1\right)} d_{a c_{1} c_{2}} d_{a c_{1}^{\prime} c_{2}^{\prime}} \operatorname{Tr}\left(T_{f, s}^{c_{1}} T_{f, s}^{c_{2}^{\prime}} T_{f, s}^{c_{2}} c_{f, s}^{c_{1}^{\prime}}+T_{f, s}^{c_{2}} T_{f, s}^{c_{2}^{\prime}} T_{f, s}^{c_{1}} T_{f, s}^{c_{1}^{\prime}}\right)
$$

they coincide with the Pomeron kernels in usual QED [20], [21] and scalar QED. It is important that these pieces are infrared finite, such that the infrared singular parts of the kernels for symmetric and antisymmetric adjoint representations are the same. Moreover, the singular parts of $K_{r}^{f}$ and $K_{r}^{d}$ are equal to the half of the infrared singular part of the colour singlet kernel: this makes the procedure of cancellation of the infrared singularities for all three kernels the same. Note that the coefficients $b_{f, s}$ are zero when the colour representations for the particles $f, s$ coincide with that for the gluons, i.e. $T_{a b}^{c}=-i f_{a b c}$ as in $N=4$ SUSY. This explains why the gluon contribution to $K_{r}^{f, d}$ is the same for both adjoint representations. We finally note that at large $N_{c}$ the nonplanar contributions do not contribute.

The contributions $K_{f}^{f}$ [4] and $K_{s}^{f}$ [19] are known at arbitrary $D$ :

$$
K_{f}^{f}\left(\vec{q}_{1}, \vec{q}_{1}^{\prime}, \vec{q}\right)=\frac{2 \bar{g}^{4} a_{f}}{N_{c} \pi^{1+\epsilon} \Gamma(1-\epsilon)} \frac{\Gamma^{2}(2+\epsilon)}{\epsilon \Gamma(4+2 \epsilon)}\left\{2\left(\frac{{\overrightarrow{q_{1}}}^{2}{\overrightarrow{q_{2}}}^{2}+{\overrightarrow{q_{1}}}^{2}{\overrightarrow{q_{2}}}^{2}}{\vec{k}^{2}}-\vec{q}^{2}\right)\left(\vec{k}^{2}\right)^{\epsilon}+\right.
$$




$$
\begin{aligned}
& +\vec{q}^{2}\left(2\left(\vec{k}^{2}\right)^{\epsilon}+2\left(\vec{q}^{2}\right)^{\epsilon}-\left(\vec{q}_{1}^{2}\right)^{\epsilon}-\left({\overrightarrow{q_{2}}}^{2}\right)^{\epsilon}-\left({\overrightarrow{q_{1}}}^{2}\right)^{\epsilon}-\left({\overrightarrow{q_{2}}}^{2}\right)^{\epsilon}\right) \\
& \left.-\left(\frac{{\overrightarrow{q_{1}}}^{2}{\overrightarrow{q_{2}}}^{\prime 2}-{\overrightarrow{q_{2}}}^{2}{\overrightarrow{q_{1}}}^{\prime 2}}{\vec{k}^{2}}\right)\left(\left(\vec{q}_{1}^{2}\right)^{\epsilon}-\left({\overrightarrow{q_{2}}}^{2}\right)^{\epsilon}-\left({\overrightarrow{q_{1}}}^{2}\right)^{\epsilon}+\left({\overrightarrow{q_{2}}}^{2}\right)^{\epsilon}\right)\right\},
\end{aligned}
$$

where the total transverse momenta in the $s$ and $t$ channel are given by

$$
\vec{k}=\vec{q}_{1}-\vec{q}_{1}^{\prime}=\vec{q}_{2}^{\prime}-\vec{q}_{2}
$$

and

$$
\vec{q}=\vec{q}_{1}+\vec{q}_{2}=\vec{q}_{1}^{\prime}+\vec{q}_{2}^{\prime}
$$

resp., and

$$
K_{s}^{f}\left(\vec{q}_{1}, \vec{q}_{1}^{\prime}, \vec{q}\right)=\frac{a_{s}}{4(1+\epsilon) a_{f}} K_{f}\left(\vec{q}_{1}, \vec{q}_{1}^{\prime}, \vec{q}\right)
$$

Turning now to $K_{r}^{f}$, in principle it is possible [5] to write the gluon contribution of the real kernel also at arbitrary $D$. But the result looks too cumbersome. With the accuracy of keeping, after integration over $\vec{k}$, all terms nonvanishing at $\epsilon \rightarrow 0$, one obtains for the total real kernel in the antisymmetric octet representation, $K_{r}^{f}$, expressed in terms of the renormalized coupling

$$
\begin{gathered}
K_{r}^{f}\left(\vec{q}_{1}, \vec{q}_{1}^{\prime}, \vec{q}\right)=\frac{\bar{g}_{\mu}^{2} \mu^{-2 \epsilon}}{\pi^{1+\epsilon} \Gamma(1-\epsilon)}\left(\frac{\vec{q}_{1}^{2} \vec{q}_{2}^{\prime 2}+\vec{q}_{1}^{\prime 2} \vec{q}_{2}^{2}}{\vec{k}^{2}}-\vec{q}^{2}\right) \\
\times\left(1+\bar{g}_{\mu}^{2}\left[\frac{\beta_{0}}{N_{c} \epsilon}+\left(\frac{\vec{k}^{2}}{\mu^{2}}\right)^{\epsilon}\left(-\frac{\beta_{0}}{N_{c} \epsilon}+\frac{67}{9}-2 \zeta(2)-\frac{10}{9} \frac{a_{f}}{N_{c}}-\frac{4}{9} \frac{a_{s}}{N_{c}}\right.\right.\right. \\
\left.\left.\left.+\epsilon\left(-\frac{404}{27}+14 \zeta(3)+\frac{\beta_{0}}{N_{c}} \zeta(2)+\frac{56}{27} \frac{a_{f}}{N_{c}}+\frac{26}{27} \frac{a_{s}}{N_{c}}\right)\right)\right]\right)+\frac{\bar{g}_{\mu}^{4}}{2 \pi} R\left(\vec{q}_{1}, \vec{q}_{1}^{\prime}, \vec{q}\right),
\end{gathered}
$$

where $\vec{k}$ is given in (15). The function $R\left(\vec{q}_{1}, \vec{q}_{1}^{\prime}, \vec{q}\right)$ is infrared safe and can be evaluated at $D=4$ :

$$
\begin{gathered}
R\left(\vec{q}_{1}, \vec{q}_{1}^{\prime}, \vec{q}\right)=\left[\vec { q } ^ { 2 } \left(\frac{\beta_{0}}{N_{c}} \ln \left(\frac{\vec{q}_{1}^{2} \vec{q}_{1}^{\prime 2}}{\vec{q}^{2} \vec{k}^{2}}\right)+\frac{1}{2} \ln \left(\frac{\vec{q}_{1}^{2}}{\vec{q}^{2}}\right) \ln \left(\frac{\vec{q}_{2}^{2}}{\vec{q}^{2}}\right)+\frac{1}{2} \ln \left(\frac{\vec{q}_{1}^{\prime 2}}{\vec{q}^{2}}\right) \ln \left(\frac{\vec{q}_{2}^{\prime 2}}{\vec{q}^{2}}\right)\right.\right. \\
\left.+\frac{1}{2} \ln ^{2}\left(\frac{\vec{q}_{1}^{2}}{\vec{q}_{1}^{\prime 2}}\right)\right)-\frac{\vec{q}_{1}^{2} \vec{q}_{2}^{\prime 2}+\vec{q}_{2}^{2} \vec{q}_{1}^{\prime 2}}{\vec{k}^{2}} \ln ^{2}\left(\frac{\vec{q}_{1}^{2}}{\vec{q}_{1}^{\prime 2}}\right)+\frac{\vec{q}_{1}^{2} \vec{q}_{2}^{\prime 2}-\vec{q}_{2}^{2} \vec{q}_{1}^{\prime 2}}{\vec{k}^{2}} \ln \left(\frac{\vec{q}_{1}^{2}}{\vec{q}_{1}^{\prime 2}}\right) \\
\times\left(\frac{\beta_{0}}{N_{c}}-\frac{1}{2} \ln \left(\frac{\vec{q}_{1}^{2} \vec{q}_{1}^{\prime 2}}{\vec{k}^{4}}\right)\right)+4 \frac{\left(\vec{k} \times \vec{q}_{1}\right)}{\vec{k}^{2}}\left(\vec{k}^{2}\left(\vec{q}_{1} \times \vec{q}_{2}\right)-\vec{q}_{1}^{2}\left(\vec{k} \times \vec{q}_{2}\right)-\vec{q}_{2}^{2}\left(\vec{k} \times \vec{q}_{1}\right)\right) I_{\vec{q}_{1},-\vec{k}} \\
+\left(\vec{q}_{1} \leftrightarrow-\vec{q}_{2}, \quad \vec{q}_{1}^{\prime} \leftrightarrow-\vec{q}_{2}^{\prime}\right) .
\end{gathered}
$$

Here $(\vec{a} \times \vec{b})=a_{x} b_{y}-a_{y} b_{x}$ and

$$
I_{\vec{p}, \vec{q}}=\int_{0}^{1} \frac{d x}{(\vec{p}+x \vec{q})^{2}} \ln \left(\frac{\vec{p}^{2}}{x^{2} \vec{q}^{2}}\right)
$$


can be expressed in terms of the di-logarithmic function and has the following symmetry properties:

$$
I_{\vec{p}, \vec{q}}=I_{-\vec{p},-\vec{q}}=I_{\vec{q}, \vec{p}}=I_{\vec{p},-\vec{p}-\vec{q}} .
$$

This symmetry is evident from the representation

$$
I_{\vec{p}, \vec{q}}=\int_{0}^{1} \int_{0}^{1} \int_{0}^{1} \frac{d x_{1} d x_{2} d x_{3} \delta\left(1-x_{1}-x_{2}-x_{3}\right)}{\left(\vec{p}^{2} x_{1}+\vec{q}^{2} x_{2}+(\vec{p}+\vec{q})^{2} x_{3}\right)\left(x_{1} x_{2}+x_{1} x_{3}+x_{2} x_{3}\right)} .
$$

Other useful representations are

$$
\begin{aligned}
I_{\vec{p}, \vec{q}} & =\int_{0}^{1} \frac{d x}{a(1-x)+b x-c x(1-x)} \ln \left(\frac{a(1-x)+b x}{c x(1-x)}\right) \\
& =\int_{0}^{1} d x \int_{0}^{1} d z \frac{1}{c x(1-x) z+(b(1-x)+a x)(1-z)},
\end{aligned}
$$

where $a=\vec{p}^{2}, b=\vec{q}^{2}, c=(\vec{p}+\vec{q})^{2}$.

Keeping only the terms of zero order in $\epsilon$ in the trajectory (44) and passing to the renormalized coupling, one obtains

$$
\omega\left(-\vec{q}_{i}^{2}\right)=-\frac{\bar{g}_{\mu}^{2} \vec{q}_{i}^{2}}{\pi^{1+\epsilon} \Gamma(1-\epsilon)} \int \frac{d^{2+2 \epsilon} k \mu^{-2 \epsilon}}{\vec{k}^{2}\left(\vec{k}-\vec{q}_{i}\right)^{2}}\left(1+\bar{g}_{\mu}^{2} f_{\mu}^{\omega}\left(\vec{k}, \vec{k}-\vec{q}_{i}\right)\right)
$$

where

$$
\begin{gathered}
f_{\mu}^{\omega}\left(\vec{k}_{1}, \vec{k}_{2}\right)=\frac{\beta_{0}}{N_{c} \epsilon}+\left[\frac{\beta_{0}}{N_{c} \epsilon}-\frac{67}{9}+2 \zeta(2)+\frac{10}{9} \frac{a_{f}}{N_{c}}\right. \\
\left.+\frac{4}{9} \frac{a_{s}}{N_{c}}+\epsilon\left(\frac{404}{27}-\frac{\beta_{0}}{N_{c}} \zeta(2)-6 \zeta(3)-\frac{56}{27} \frac{a_{f}}{N_{c}}-\frac{26}{27} \frac{a_{s}}{N_{c}}\right)\right] \\
\times\left[\left(\frac{\vec{k}_{12}^{2}}{\mu^{2}}\right)^{\epsilon}-\left(\frac{\vec{k}_{1}^{2}}{\mu^{2}}\right)^{\epsilon}-\left(\frac{\vec{k}_{2}^{2}}{\mu^{2}}\right)^{\epsilon}\right]-\ln \left(\frac{\vec{k}_{12}^{2}}{\vec{k}_{1}^{2}}\right) \ln \left(\frac{\vec{k}_{12}^{2}}{\vec{k}_{2}^{2}}\right) .
\end{gathered}
$$

This representation allows to calculate $\omega\left(-\vec{q}^{2}\right)$ explicitly:

$$
\begin{aligned}
\omega\left(-\vec{q}^{2}\right)= & -\bar{g}_{\mu}^{2}\left(\frac{2}{\epsilon}+2 \ln \left(\frac{\vec{q}^{2}}{\mu^{2}}\right)\right)-\bar{g}_{\mu}^{4}\left[\frac{\beta_{0}}{N_{c}}\left(\frac{1}{\epsilon^{2}}-\ln ^{2}\left(\frac{\vec{q}^{2}}{\mu^{2}}\right)\right)+\left(\frac{1}{\epsilon}+2 \ln \left(\frac{\vec{q}^{2}}{\mu^{2}}\right)\right)\right. \\
& \left.\times\left(\frac{67}{9}-2 \zeta(2)-\frac{10}{9} \frac{a_{f}}{N_{c}}-\frac{4}{9} \frac{a_{s}}{N_{c}}\right)-\frac{404}{27}+2 \zeta(3)+\frac{56}{27} \frac{a_{f}}{N_{c}}+\frac{26}{27} \frac{a_{s}}{N_{c}}\right] .
\end{aligned}
$$

In order to cancel the infrared singularities and to find the kernel (3) at $D=4$ let us introduce the cut-off $\lambda \rightarrow 0$ which should be taken to zero after pushing $\epsilon \rightarrow 0$. In the integral representation of the trajectory (24) we divide the integration region into three domains. In two of them either $\vec{k}^{2} \leq \lambda^{2}$, or $\left(\vec{k}-\vec{q}_{i}\right)^{2} \leq \lambda^{2}$, and in the third one both $\vec{k}^{2}>\lambda^{2}$ and $\left(\vec{k}-\vec{q}_{i}\right)^{2}>\lambda^{2}$. Then in the third domain we can take $\epsilon=0$ in (25) and put $f_{\mu}^{\omega}\left(\vec{k}_{1}, \vec{k}_{2}\right)=f_{\omega}^{(0)}\left(\vec{k}_{1}, \vec{k}_{2}\right)$, where

$$
f_{\omega}^{(0)}\left(\vec{k}_{1}, \vec{k}_{2}\right)=\frac{67}{9}-2 \zeta(2)-\frac{10}{9} \frac{a_{f}}{N_{c}}-\frac{4}{9} \frac{a_{s}}{N_{c}}-\frac{\beta_{0}}{N_{c}} \ln \left(\frac{\vec{k}_{1}^{2} \vec{k}_{2}^{2}}{\mu^{2} \vec{k}_{12}^{2}}\right)-\ln \left(\frac{\vec{k}_{12}^{2}}{\vec{k}_{1}^{2}}\right) \ln \left(\frac{\vec{k}_{12}^{2}}{\vec{k}_{2}^{2}}\right) .
$$


In the first domain we have

$$
\begin{gathered}
f_{\mu}^{\omega}\left(\vec{k}, \vec{k}-\vec{q}_{i}\right)=\frac{\beta_{0}}{N_{c} \epsilon}-\left(\frac{\vec{k}^{2}}{\mu^{2}}\right)^{\epsilon}\left[\frac{\beta_{0}}{N_{c} \epsilon}-\frac{67}{9}+2 \zeta(2)+\frac{10}{9} \frac{a_{f}}{N_{c}}+\frac{4}{9} \frac{a_{s}}{N_{c}}\right. \\
\left.+\epsilon\left(\frac{404}{27}-\frac{\beta_{0}}{N_{c}} \zeta(2)-6 \zeta(3)-\frac{56}{27} \frac{a_{f}}{N_{c}}-\frac{26}{27} \frac{a_{s}}{N_{c}}\right)\right],
\end{gathered}
$$

and in the second one we have the same expression with the substitution $\vec{k}^{2} \rightarrow\left(\vec{k}-\vec{q}_{i}\right)^{2}$.

Writing the real part of the antisymmetric color octet kernel as

$$
K_{r}^{f}\left(\vec{q}_{1}, \vec{q}_{1}^{\prime}, \vec{q}\right)=K_{r}^{f}\left(\vec{q}_{1}, \vec{q}_{1}^{\prime}, \vec{q}\right) \theta\left(\lambda^{2}-\vec{k}^{2}\right)+K_{r}^{f}\left(\vec{q}_{1}, \vec{q}_{1}^{\prime}, \vec{q}\right) \theta\left(\vec{k}^{2}-\lambda^{2}\right),
$$

and comparing (18) with (28), we see that in the kernel $K_{12}^{f}(\underline{31})$ the first term in the rhs of (29) cancels almost completely against the contributions of the regions $\vec{k}^{2} \leq \lambda^{2}$ and $\left(\vec{k}-\vec{q}_{i}\right)^{2} \leq \lambda^{2}$ in the trajectories $\omega\left(-\vec{q}_{i}^{2}\right)$. The only piece which remains uncanceled in each of the trajectories for $\epsilon \rightarrow 0$ is

$$
\frac{\bar{g}_{\mu}^{4}}{\pi^{1+\epsilon} \Gamma(1-\epsilon)} \int \frac{d^{2+2 \epsilon} k \mu^{-2 \epsilon}}{\vec{k}^{2}} 8 \epsilon \zeta(3)\left(\frac{\vec{k}^{2}}{\mu^{2}}\right)^{\epsilon} \theta\left(\lambda^{2}-\vec{k}^{2}\right)=\frac{\alpha_{s}^{2}(\mu) N_{c}^{2}}{4 \pi^{2}} \zeta(3) .
$$

On account of this cancellation and using the equality

$$
\int \frac{d^{2} k}{4 \pi} \frac{\vec{q}^{2}}{\vec{k}^{2}(\vec{k}-\vec{q})^{2}} \ln \left(\frac{\vec{k}^{2}}{\vec{q}^{2}}\right) \ln \left(\frac{(\vec{k}-\vec{q})^{2}}{\vec{q}^{2}}\right)=\zeta(3)
$$

we can obtain

$$
\begin{gathered}
K_{12}^{f}=-\delta\left(\vec{q}_{1}-\vec{q}_{1}^{\prime}\right) \vec{q}_{1}^{2} \vec{q}_{2}^{2} \frac{\alpha_{s}(\mu) N_{c}}{8 \pi^{2}}\left(\int d ^ { 2 } k \left[\frac{2}{\vec{k}^{2}}+2 \frac{\vec{k}\left(\vec{q}_{1}-\vec{k}\right)}{\vec{k}^{2}\left(\vec{q}_{1}-\vec{k}\right)^{2}}\right.\right. \\
\left.\left.+\frac{\alpha_{s}(\mu) N_{c}}{\pi}\left(V(\vec{k})+V\left(\vec{k}, \vec{k}-\vec{q}_{1}\right)\right)\right]-3 \alpha_{s}(\mu) N_{c} \zeta(3)\right) \\
+\frac{\alpha_{s}(\mu) N_{c}}{8 \pi^{2}}\left\{( \frac { \vec { q } _ { 1 } ^ { 2 } \vec { q } _ { 2 } ^ { \prime 2 } + \vec { q } _ { 1 } ^ { \prime 2 } \vec { q } _ { 2 } ^ { 2 } } { \vec { k } ^ { 2 } } - \vec { q } ^ { 2 } ) \left(1+\frac{\alpha_{s}(\mu) N_{c}}{4 \pi}\left[-\frac{\beta_{0}}{N_{c}} \ln \left(\frac{\vec{k}^{2}}{\mu^{2}}\right)+\frac{67}{9}\right.\right.\right. \\
\left.\left.\left.-2 \zeta(2)-\frac{10}{9} \frac{a_{f}}{N_{c}}-\frac{4}{9} \frac{a_{s}}{N_{c}}\right]\right)\right\}+\frac{\alpha_{s}^{2}(\mu) N_{c}^{2}}{4 \pi} R\left(\vec{q}_{1}, \vec{q}_{1}^{\prime}, \vec{q}\right)+\left(\vec{q}_{1} \leftrightarrow-\vec{q}_{2}, \quad \vec{q}_{1}^{\prime} \leftrightarrow-\vec{q}_{2}^{\prime}\right),
\end{gathered}
$$

where

$$
\begin{gathered}
V(\vec{k})=\frac{1}{2 \vec{k}^{2}}\left(\frac{67}{9}-2 \zeta(2)-\frac{10}{9} \frac{a_{f}}{N_{c}}-\frac{4}{9} \frac{a_{s}}{N_{c}}-\frac{\beta_{0}}{N_{c}} \ln \left(\frac{\vec{k}^{2}}{\mu^{2}}\right)\right), \\
V(\vec{k}, \vec{q})=\frac{\vec{k} \vec{q}}{2 \vec{k}^{2} \vec{q}^{2}}\left(\frac{\beta_{0}}{N_{c}} \ln \left(\frac{\vec{k}^{2} \vec{q}^{2}}{\mu^{2}(\vec{k}-\vec{q})^{2}}\right)-\frac{67}{9}+2 \zeta(2)+\frac{10}{9} \frac{a_{f}}{N_{c}}+\frac{4}{9} \frac{a_{s}}{N_{c}}\right)
\end{gathered}
$$




$$
-\frac{\beta_{0}}{4 N_{c} \vec{k}^{2}} \ln \left(\frac{\vec{q}^{2}}{(\vec{k}-\vec{q})^{2}}\right)-\frac{\beta_{0}}{4 N_{c} \vec{q}^{2}} \ln \left(\frac{\vec{k}^{2}}{(\vec{k}-\vec{q})^{2}}\right) .
$$

Of course, the infrared singularities in (32) must be regularized either by limitations on the integration regions as discussed above or in an equivalent way.

Thus we have obtained the antisymmetric octet kernel, $K_{12}^{f}$, at $D=4$. For the Odderon which belongs to the symmetric representation ( $d$-coupling), the kernel $K_{12}^{d}$ differs from $K_{12}^{f}$ only by the abelian parts of the fermion and scalar contributions,

$$
K_{12}^{d}=K_{12}^{f}+K_{f}^{a}+K_{s}^{a},
$$

which have no infrared singularities and can directly be taken at $D=4$. However, the corresponding expressions are rather complicated. The fact that for the antisymmetric adjoint representation the kernel was relatively simple is connected with the gluon reggeization and with the absence of the contribution of non-planar diagrams in this kernel. In contrast to this, the abelian parts contain such nonplanar contributions (crossed fermion and scalar loops).

From the results of [4] and [22] it follows:

$$
\begin{gathered}
K_{f}^{a}\left(\vec{q}_{1}, \vec{q}_{1}^{\prime}, \vec{q}\right)=\frac{\alpha_{s}^{2}(\mu) b_{f}}{4 \pi^{3}} \int_{0}^{1} d x \int \frac{d^{2} l_{1}}{\pi}\left[2 x(1-x) \frac{2\left(\vec{q}_{1} \vec{l}_{1}\right)-\vec{q}_{1}^{2}}{\sigma_{11}}\left(\frac{2\left(\vec{q}_{2} \vec{l}_{1}\right)+\vec{q}_{2}^{2}}{\sigma_{12}}+\frac{2\left(\vec{q}_{2} \vec{l}_{2}\right)+\vec{q}_{2}^{2}}{\sigma_{22}}\right)\right. \\
+\frac{x \vec{q}^{2}\left(\vec{q}_{1}^{2}-2\left(\vec{q}_{1} \vec{l}_{1}\right)\right)}{\sigma_{11} \sigma_{12}}+\frac{1}{\sigma_{11} \sigma_{22}}\left(-4\left(\vec{q}_{1} \vec{l}_{1}\right)\left(\vec{q}_{2} \vec{q}_{2}^{\prime}\right)-2\left(\overrightarrow{l_{1}} \vec{k}\right) \vec{q}_{1}^{2}+2\left(\overrightarrow{q_{1}} \vec{k}\right)\left(\overrightarrow{q_{2}} \vec{k}\right)+\vec{q}_{1}^{2} \vec{q}_{2}^{2}\right. \\
\left.\left.+\frac{\vec{k}^{2}\left(\vec{q}_{1}-\vec{q}_{2}\right)^{2}}{2}+x \vec{q}^{2}\left(2\left(\vec{q}_{1} \vec{l}_{1}\right)-\vec{q}_{1}^{2}\right)\right)\right]+\left(\vec{q}_{1} \leftrightarrow \vec{q}_{2}, \vec{q}_{1}^{\prime} \leftrightarrow \vec{q}_{2}^{\prime}\right),
\end{gathered}
$$

where

$$
\begin{gathered}
\overrightarrow{l_{1}}+\overrightarrow{l_{2}}=\vec{k}=\vec{q}_{1}-\vec{q}_{1}^{\prime}=\vec{q}_{2}^{\prime}-\vec{q}_{2}, \\
\sigma_{11}=\left(\vec{l}_{1}-x \vec{q}_{1}\right)^{2}+x(1-x) \vec{q}_{1}^{2}, \quad \sigma_{12}=\left(\vec{l}_{1}+x \vec{q}_{2}\right)^{2}+x(1-x) \vec{q}_{2}^{2}, \\
\sigma_{22}=\left(\overrightarrow{l_{2}}+(1-x) \vec{q}_{2}\right)^{2}+x(1-x) \vec{q}_{2}^{2} .
\end{gathered}
$$

Performing the integration over $\vec{l}_{1}$ with the help of Feynman parametrization, we obtain

$$
\begin{gathered}
K_{f}^{a}\left(\vec{q}_{1}, \vec{q}_{1}^{\prime}, \vec{q}\right)=\frac{\alpha_{s}^{2}(\mu) b_{f}}{4 \pi^{3}} \int_{0}^{1} d x \int_{0}^{1} d z\left[-2 x(1-x)\left(2 \vec{q}_{1} \vec{q}_{2} \ln \left(\frac{R_{11}}{R_{12}}\right)\right.\right. \\
\left.+\frac{\left(\vec{q}_{1}^{2}-2 \vec{r}_{11} \vec{q}_{1}\right)\left(\vec{q}_{2}^{2}+2 \vec{r}_{11} \vec{q}_{2}\right)}{R_{11}}+\frac{\left(\vec{q}_{1}^{2}-2 \vec{r}_{12} \vec{q}_{1}\right)\left(\vec{q}_{2}^{\prime 2}-\vec{k}^{2}-2 \vec{r}_{12} \vec{q}_{2}\right)}{R_{12}}\right) \\
+\frac{x \vec{q}^{2}\left(\vec{q}_{1}^{2}-2 \vec{r}_{11} \vec{q}_{1}\right)}{R_{11}}+\frac{1}{R_{12}}\left(-4\left(\vec{q}_{2} \vec{q}_{2}^{\prime}\right)\left(\vec{q}_{1} \vec{r}_{12}\right)-2 \vec{q}_{1}^{2}\left(\vec{r}_{12} \vec{k}\right)+2\left(\overrightarrow{q_{1}} \vec{k}\right)\left(\vec{q}_{2} \vec{k}\right)+\vec{q}_{1}^{2} \vec{q}_{2}^{2}\right. \\
\left.\left.+\frac{\vec{k}^{2}\left(\vec{q}_{1}-\vec{q}_{2}\right)^{2}}{2}+x \vec{q}^{2}\left(2\left(\vec{q}_{1} \vec{r}_{12}\right)-\vec{q}_{1}^{2}\right)\right)\right]+\left(\vec{q}_{1} \leftrightarrow \vec{q}_{2}, \vec{q}_{1}^{\prime} \leftrightarrow \vec{q}_{2}^{\prime}\right),
\end{gathered}
$$


where

$$
\begin{gathered}
\vec{r}_{11}=x\left(z \vec{q}_{1}-(1-z) \vec{q}_{2}\right), \vec{r}_{12}=x z \vec{q}_{1}+(1-x)(1-z) \vec{q}_{2}+(1-z) \vec{k}, \\
R_{11}=x(1-x)\left(z \vec{q}_{1}^{2}+(1-z) \vec{q}_{2}^{2}\right)+x^{2} z(1-z) \vec{q}^{2}, \\
R_{12}=x(1-x)\left(z \vec{q}_{1}^{2}+(1-z) \vec{q}_{2}^{2}\right)+z(1-z)\left(x \vec{q}_{1}^{\prime 2}+(1-x) \vec{q}_{2}^{\prime 2}-x(1-x) \vec{q}^{2}\right) .
\end{gathered}
$$

Further integration leads to a lengthy expression which will not be presented here.

For the scalar contribution we have, using the results of [19]:

$$
\begin{aligned}
& K_{s}^{a}\left(\vec{q}_{1}, \vec{q}_{1}^{\prime}, \vec{q}\right)=\frac{\alpha_{s}^{2}(\mu) b_{s}}{4 \pi^{3}} \int_{0}^{1} d x \int \frac{d \vec{l}_{1}}{\pi} x(1-x) \frac{{\overrightarrow{q_{1}}}^{2}-2\left(\overrightarrow{l_{1}} \overrightarrow{q_{1}}\right)}{\sigma_{11}} \\
& \times\left(\frac{{\overrightarrow{q_{2}}}^{2}+2\left(\overrightarrow{l_{1}} \overrightarrow{q_{2}}\right)}{\sigma_{12}}+\frac{{\overrightarrow{q_{2}}}^{2}+2\left(\overrightarrow{l_{2}} \overrightarrow{q_{2}}\right)}{\sigma_{22}}\right)+\left(\overrightarrow{q_{1}} \leftrightarrow \vec{q}_{2}, \vec{q}_{1}^{\prime} \leftrightarrow \vec{q}_{2}^{\prime}\right),
\end{aligned}
$$

and after integration over $\vec{l}_{1}$

$$
\begin{gathered}
K_{s}^{a}\left(\vec{q}_{1}, \vec{q}_{1}^{\prime}, \vec{q}\right)=\frac{\alpha_{s}^{2}(\mu) b_{s}}{2 \pi^{3}} \int_{0}^{1} d x x(1-x) \int_{0}^{1} d z\left[2 \vec{q}_{1} \vec{q}_{2} \ln \left(\frac{R_{11}}{R_{12}}\right)\right. \\
\left.+\frac{\left(\vec{q}_{1}^{2}-2 \vec{r}_{11} \vec{q}_{1}\right)\left(\vec{q}_{2}^{2}+2 \vec{r}_{11} \vec{q}_{2}\right)}{R_{11}}+\frac{\left(\vec{q}_{1}^{2}-2 \vec{r}_{12} \vec{q}_{1}\right)\left(\vec{q}_{2}^{\prime 2}-\vec{k}^{2}-2 \vec{r}_{12} \vec{q}_{2}\right)}{R_{12}}\right] .
\end{gathered}
$$

The abelian contributions are drastically simplified if we transform them into the space of transverse coordinates and choose the Möbius representation [23. The Möbius form of these contributions can be written [24] as

$$
\begin{aligned}
\left\langle\vec{r}_{1} \vec{r}_{2}\left|\hat{\mathcal{K}}_{M}^{a}\right| \vec{r}_{1}^{\prime} \vec{r}_{2}^{\prime}\right\rangle=\frac{\alpha_{s}^{2}}{2 \pi^{4}} & \frac{1}{\vec{r}_{1^{\prime} 2^{\prime}}^{4}}\left[\left(\frac{\vec{r}_{11^{\prime}}^{2} \vec{r}_{22^{\prime}}^{2}-2 \vec{r}_{12}^{2} \vec{r}_{1^{\prime} 2^{\prime}}^{2}}{d} \ln \left(\frac{\vec{r}_{12^{\prime}}^{2} \vec{r}_{21^{\prime}}^{2}}{\vec{r}_{11^{\prime}}^{2} \vec{r}_{22^{\prime}}^{2}}\right)-1\right)\left(b_{s}-2 b_{f}\right)\right. \\
& \left.+\frac{\left(2 b_{s}-3 b_{f}\right)}{\vec{r}_{1^{\prime} 2^{\prime}}^{2}} \frac{\vec{r}_{12}^{2}}{d} \ln \left(\frac{\vec{r}_{12^{\prime}}^{2}}{\vec{r}_{11^{\prime}}^{2} \vec{r}_{22^{\prime}}^{2}}\right)\right] .
\end{aligned}
$$

Here $\vec{r}_{i j^{\prime}}=\vec{r}_{i}-\vec{r}_{j}^{\prime}$.

Finally, the total $2 \rightarrow 2$ kernel (2) for the Odderon is expressed in terms of $K_{i j}^{d}$. It is worthwhile to note here that the real part of the kernel turns to zero at zero transverse momentum of any of the reggeons.

\section{The $3 \rightarrow 3$ kernel}

The kinematics of the lowest order $3 \rightarrow 3$ transition is illustrated in Fig.2. The grey blobs on the right and on the left hand side are impact factors for the external projectiles $A$, and $B$, the horizontal wavy lines denote reggeized gluons, and the black ellipse in the center stands for the $3 \rightarrow 3$ vertex we are interested in. 


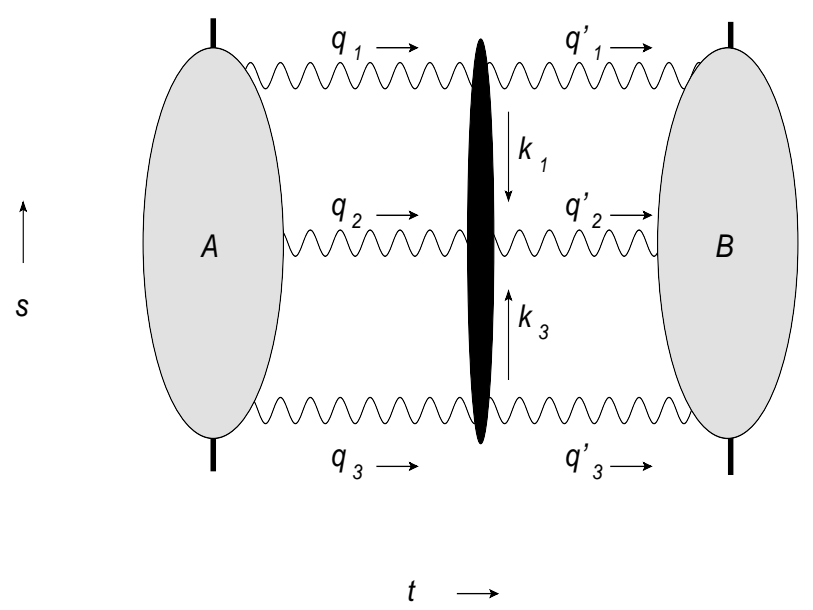

Fig.2: Kinematics of the $3 \rightarrow 3$ transition of reggeized gluons.

In this section the derivation of this connected $3 \rightarrow 3$ vertex in lowest order will be described in some detail. We make use of the effective action [13, 14] and of the Ward identities discussed in [15].

We introduce the Sudakov decomposition of the reggeon momenta

$$
q_{i}^{\mu}=\frac{1}{2}\left(n^{-}\right)^{\mu} q_{i}^{+}+\frac{1}{2}\left(n^{+}\right)^{\mu} q_{i}^{-}+q_{i \perp}^{\mu},
$$

where the lightlike vectors $n^{-}$and $n^{+}$have the property

$$
\left(n^{-}\right)^{2}=\left(n^{+}\right)^{2}=0, n^{-} n^{+}=2 .
$$

The gluon vertices and diagrams we need to include 1 are illustrated in Fig.3 which shows the building blocks derived form the effective action in [14: (a) the effective RPRproduction vertex, (b) the PPR vertex, (c) the RPRR, and (d) the RRPR, and (e) the RPPR vertex. It is important to emphasize that the gluons corresponding to the vertical $s$-channel lines are allowed to be off-shell. In Fig. 4 we illustrate the full $3 \rightarrow 3$ kernel, built from the effective vertices in Fig.3. In addition, we have to sum over all permutations of the reggeized gluons: in order to avoid double counting it is enough to add, on the lhs of the kernel, the three sequences (123), (231), and (312) of the lines $q_{1}, q_{2}$, and $q_{3}$ (counting from the top to the bottom). On the rhs, we sum over all six permutations of the lines $q_{1}^{\prime}, q_{2}^{\prime}$ and $q_{3}^{\prime}$. As indicated in Fig.2, in the context of the odderon kernel the $3 \rightarrow 3$ vertex will be convoluted with impact factors on both sides. These impact factors depend upon the transverse momenta; the one on the rhs contains the integrals over the longitudinal components $q_{i}^{\prime+}$, the one on the lhs is integrated over the $q_{i}{ }^{-}$. As functions of color and of the transverse momenta, the impact factors are symmetric under the exchange of gluon lines. In the case of the odderon, they are proportional to the symmetric structure constants $d_{a b c}$. When contracted with the color tensors of the $3 \rightarrow 3$ vertex, the nonplanar 3rd term in Fig.3e does not contribute. The nonplanar contribution also drops out if we insert the $3 \rightarrow 3$ vertex into the BKP evolution with overall vacuum quantum numbers and take the limit of large $N_{c}$.

\footnotetext{
${ }^{1}$ We remind that in this paper we restrict ourselves to 3 -gluon states belonging to the symmetric color representation. We therefore do not include diagrams which, for example, are antisymmetric in the pair of gluons ' 1 ' and '2'.
} 


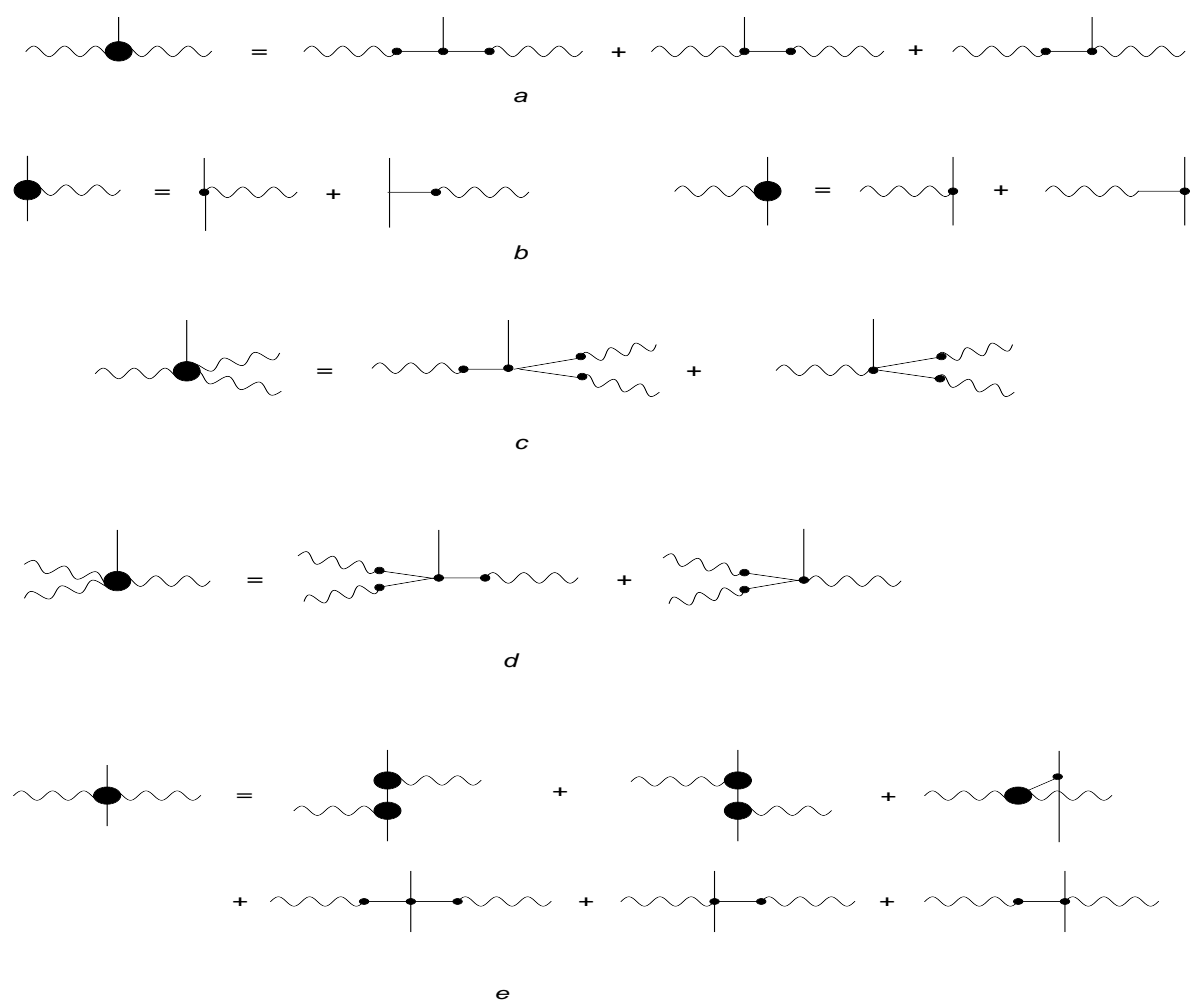

Fig.3: Building blocks derived from the effective action. The straight lines denote elementary gluons, the wavy lines correspond to reggeized gluons. Small dots stand for elementary vertices contained in the effective action, big blobs for effective vertices.

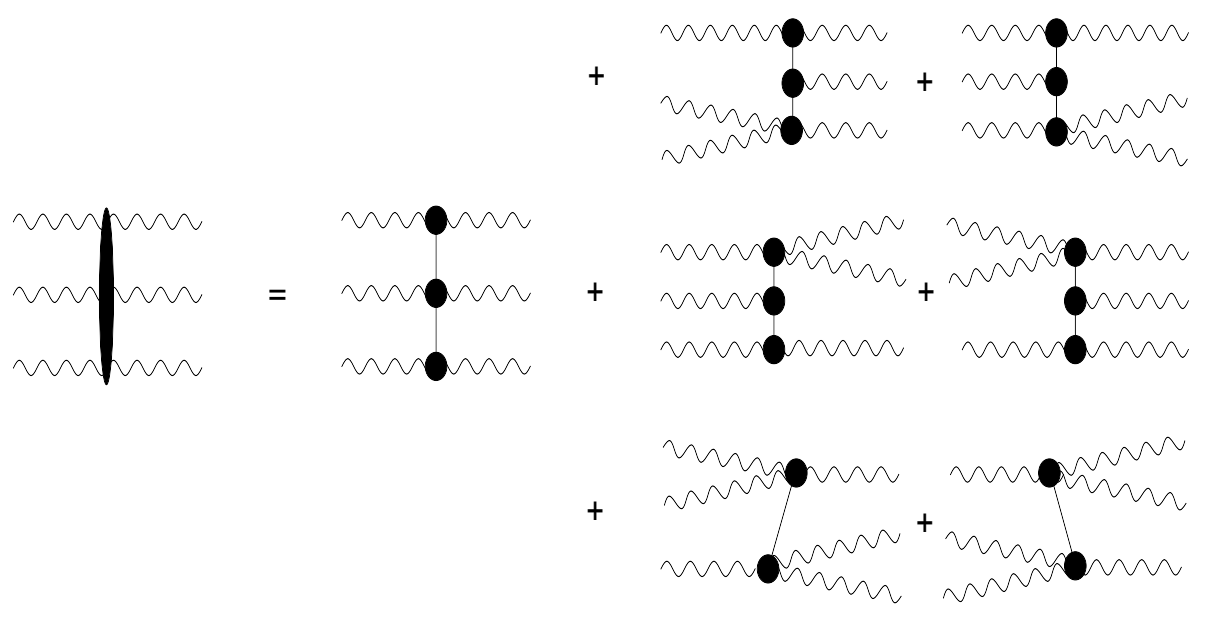

Fig.4: The full $3 \rightarrow 3$ transition kernel.

\subsection{The longitudinal integrations}

Our goal is the derivation of a closed expression for the $3 \rightarrow 3$ transition. We begin with the longitudinal integrations. Postponing the integrations over the $q^{+}$components, we first 
consider, at fixed values of $q_{1}^{+}$and $q_{3}^{+}$, the integrations over the longitudinal components $q_{1}^{\prime-}$ and $q_{3}^{\prime-}$. Ignoring, for the moment, the question whether these integrations are convergent, one might be tempted to do the integrals by picking up poles of the $s$-channel gluon propagators. To be definite, let us consider the first diagram on the rhs of Fig.4, and for the RPPR vertex in the center insert the first term on the rhs of Fig.3e. A quick look at the three $s$-channel propagators then shows that, for equal signs of $q_{1}^{+}$and $q_{3}^{+}$, the poles of the $q_{3}^{\prime-}$ integration lie on opposite sides of the integration contour, and we may pick the pole of the $k_{3}$ propagator (for the $q_{1}^{\prime-}$ integration we pick the pole of the $k_{1}$ propagator). In contrast, for opposite signs of $q_{1}^{+}$and $q_{3}^{+}$, the two poles in $q_{3}^{\prime-}$ are on the same side of the integration contour, and the integration gives zero.

In following we will prove that this simple picture, in fact, is correct. In a careful study of the longitudinal integrations we will show that only the propagator poles of the upper and lower vertical $s$-channel gluons with momenta $k_{1}$ and $k_{3}$ contribute, i.e. these gluons are on-shell. Since, on the rhs in Fig.4, only the first diagram has poles in both $k_{1}$ and $k_{3}$ : we need to compute only this diagram. It consists of the product of the effective RPR production vertices above and below with the RPPR vertex in the center. In addition we will show that, in order to obtain a nonzero result, the $q^{+}$variables have to be both positive or both negative. In deriving these results, our main problem turns out to be the convergence of the longitudinal integrations.

It is natural to start from the expressions derived from the effective action. However, in order to obtain the desired analytic expression, it is more convenient to switch to a slightly different representation. Indeed, when addressing the integrations over the longitudinal components $q_{1}^{\prime-}$ and $q_{3}^{\prime-}$ we immediately face the problem that, for the sum of the graphs shown in Fig.4, the integrals are divergent in the ultraviolet region. When taking the sum over all permutations we expect these divergences to cancel. However, in practice the computation of the sum over all permutations is rather complicated. We will circumvent this problem by making use of the Ward identities derived in [15].

In [15] it has been shown that amplitudes with reggeized gluons satisfy Ward identities. These identities can be used to replace the unphysical polarization vectors $n^{+}$and $n^{-}$ by transverse momentum vectors: for each reggeized gluon with momentum $q_{i}$, $q_{i}^{\prime}$ we substitute

$$
n^{-} \rightarrow-\frac{2}{q_{i}^{+}} q_{i \perp}, n^{+} \rightarrow-\frac{2}{q_{i}^{\prime-}} q_{i \perp}^{\prime}
$$

This leads to the appearance of additional factors $1 / q^{+}$or $1 / q^{-}$, improving the convergence of the integrals over longitudinal momenta. Special care has to be taken of the induced terms; details have been given in [15]. We apply this procedure to the sum of all diagrams of Fig.4. In addition, we include the permutations over the reggeized gluons. As a result, we strip off the contributions containing induced vertices and multiply, in the remaining QCD couplings, all horizontal lines with the corresponding transverse momenta (46). For the lines with momenta $q_{2}$ and $q_{2}^{\prime}$ we add the induced vertices. The result is illustrated in Fig.5 (we suppress the permutations; we also do not show the set of diagrams obtained by left-right reflection). In the quartic couplings on the rhs of Fig.5, we include only those color structures which match the color structures of the first two terms above and below. 


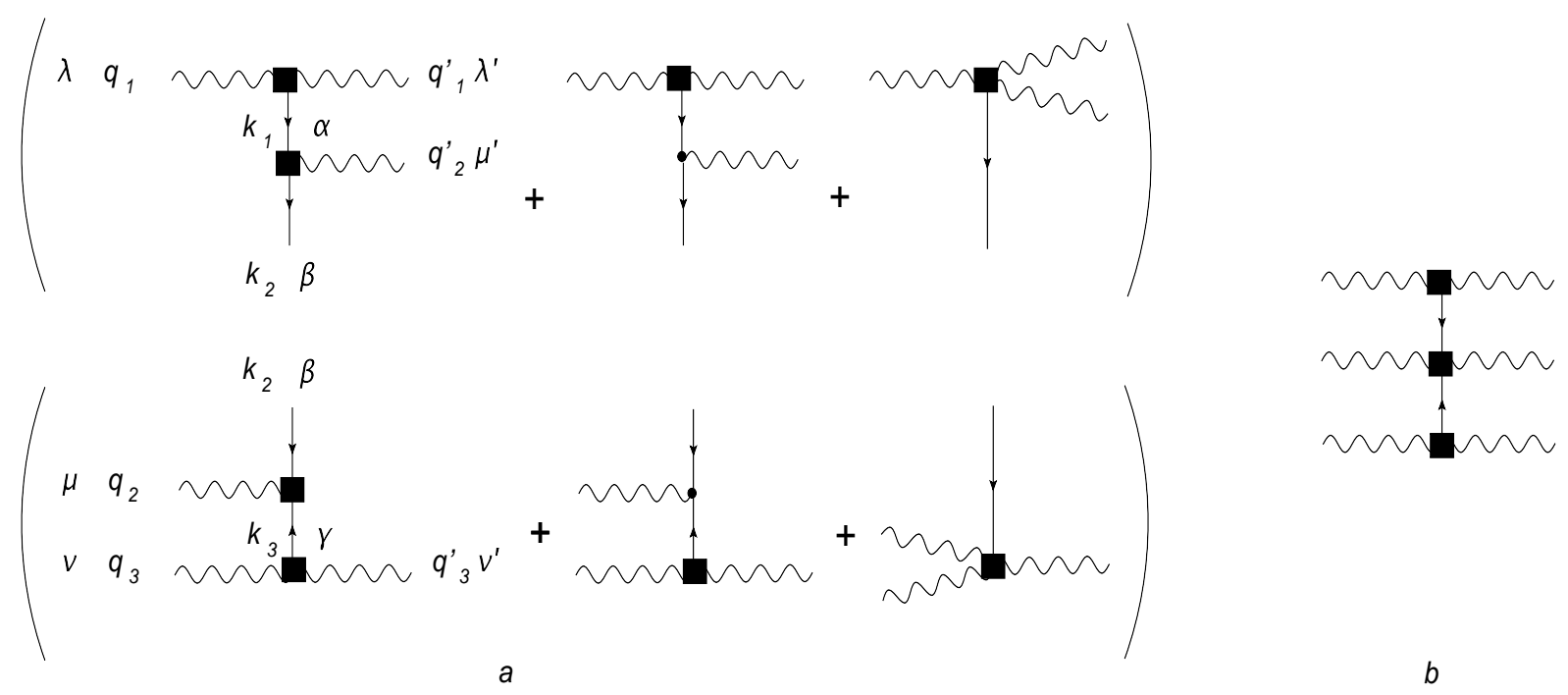

Fig.5: The $3 \rightarrow 3$ kernel. Boxes denote QCD triple (quartic) gluon vertices with transverse momentum contractions on the horizontal reggeon lines, small dots stand for induced vertices.

We stress that the Ward identities are valid only for the sum of all diagrams. Compared to the original expressions - the sum of the diagrams in Fig.4 (including the permutations of the reggeized gluons and the left-right reflected counterparts) this use of the Ward identities is equivalent to a re-grouping of contributions. After this we again consider individual sequences of reggeons, beginning with the planar structure (123).

In the first step we show that the integrations over $q_{1}^{\prime-}$ and $q_{3}^{\prime-}$ are ultraviolet convergent (at fixed transverse momenta). To this end we investigate the numerator of the sum of contributions illustrated in Fig.5. Its derivation and the final expressions are presented in the appendix. Here we only summarize those features which are essential for our discussion.

The sum of the contributions shown in Fig.5. is of the form:

$$
\frac{1}{q_{1}^{+} q_{2}^{+} q_{3}^{+} q_{1}^{\prime-} q_{2}^{\prime-} q_{3}^{\prime-}} \frac{N}{D_{1} D_{2} D_{3}}
$$

with the denominators of the three $s$-channel propagators

$$
\begin{aligned}
& D_{1}=k_{1}^{2}+i \epsilon=q_{1}{ }^{+}\left(-q_{1}^{\prime}\right)^{-}+k_{1 \perp}^{2}+i \epsilon \\
& D_{2}=k_{2}^{2}+i \epsilon=q_{1}{ }^{+} q_{3}^{\prime-}+k_{2 \perp}^{2}+i \epsilon \\
& D_{3}=k_{3}^{2}+i \epsilon=q_{3}{ }^{+}\left(-q_{3}^{\prime}\right)^{-}+k_{3 \perp}^{2}+i \epsilon .
\end{aligned}
$$

The numerator $N$ is a function of all longitudinal components $q_{i}^{+}, q_{i}^{\prime-}$, and the transverse momenta $q_{i \perp}, q_{i \perp}^{\prime}(i=1,2,3)$. Momentum conservation implies that $q_{1}^{+}+q_{2}^{+}+q_{3}^{+}=0$ and $q_{1}^{\prime-}+q_{2}^{\prime-}+q_{3}^{\prime-}=0$. In (47), the product of longitudinal components in the denominator results from the use of the Ward identities.

From the results presented in the appendix we conclude that the numerator $N$ can be written as the sum of two groups of terms:

$$
N=\sum_{(i j)(k l)} q_{i}^{+} q_{j}^{+} q_{k}^{\prime-} q_{l}^{\prime-} N_{(i j)(k l)}^{(1)}+\sum_{(i j)} q_{i}^{+} q_{j}^{\prime-} N_{(i k)}^{(2)},
$$


where the functions $N_{(i j)(k l)}^{(1)}$ and $N_{(i k)}^{(2)}$ depend upon the transverse momenta only. They have the important property that they vanish as any of the six transverse momenta $q_{i \perp}$, $q_{i \perp}^{\prime}$ goes to zero. To investigate the ultraviolet convergence of the integrations in $q_{1}^{\prime-}$ and $q_{3}^{\prime-}$, we have to combine the numerator (51) with the denominator and count the net powers of $q_{1}^{\prime-}$ and $q_{3}^{\prime-}$. Beginning in (51) with the first group (with two powers of the $q_{i}^{\prime-}$ in the numerator), we note that all but three pairings have (at least) two powers in $q_{1}^{\prime-}$ and $q_{3}^{\prime-}$ in the denominator, either from the prefactor or from the propagators $D_{i}$. It is important to note that all 'dangerous' terms in (51), e.g. terms proportional to $q_{1}^{\prime-} q_{2}^{\prime-}$, are found to be absent. The second group in (51) with only one power of $q_{i}^{\prime-}$ is safe: there are always enough powers in the denominator to provide the convergence in the ultraviolet region.

In the next step we investigate the infrared region of the integrations over $q_{1}^{\prime-}$ and $q_{3}^{\prime-}$. At first sight one might think that there are poles at $q_{i}^{\prime-}=0$ which need to be regularized. However, these poles are spurious and cancel, once we take the sum of the six permutations over the reggeized gluons on the rhs (for any fixed sequence of gluons on the lhs) and perform the integration over the $q^{+}$components. To see this in detail, we make use of the fact that the impact factors in Fig. 2 are symmetric under the exchange of the momenta of the reggeized gluons. This implies that in (47) the transverse momenta inside the numerator $N$ and the denominators $D_{i}$ can be written in the same form for all permutations, and the permutations only differ in their dependence upon the longitudinal components. It is then straightforward to show that the poles at $q_{i}^{\prime-}=0$ have zero residues. As an example, in the first group of (51) consider the term

$$
\int \frac{d q_{1}^{\prime-} d q_{3}^{\prime-}}{q_{1}^{\prime-}} \int \frac{d q_{1}^{+} d q_{3}^{+}}{q_{1}^{+}} \frac{1}{D_{1} D_{2} D_{3}} .
$$

We add the permutation with gluon "2" and gluon "3" being interchanged and use the explicit form of the propagators:

$$
\begin{gathered}
\int \frac{d q_{1}^{\prime-} d q_{3}^{\prime-}}{q_{1}^{\prime-}} . \\
\int \frac{d q_{1}^{+} d q_{3}^{+}}{q_{1}^{+}} \frac{1}{\left.q_{1}^{+}\left(-q_{1}^{\prime}\right)^{-}+k_{1 \perp}^{2}\right)}\left(\frac{1}{\left(q_{1}^{+} q_{3}^{\prime-}+k_{2 \perp}^{2}\right)\left(q_{3}{ }^{+}\left(-q_{3}^{\prime}\right)^{-}+k_{3 \perp}^{2}\right)}\right. \\
\left.+\frac{1}{\left(q_{1}^{+} q_{2}^{\prime-}+k_{2 \perp}^{2}\right)\left(q_{3}{ }^{+}\left(-q_{2}^{\prime}\right)^{-}+k_{3 \perp}^{2}\right)}\right) .
\end{gathered}
$$

In the limit $q_{1}^{\prime-} \rightarrow 0$ the sum of the second and third lines vanishes: for $q_{1}^{\prime-} \rightarrow 0$ we have $q_{2}^{\prime-} \rightarrow-q_{3}^{\prime-}$, and by the change $q_{1}^{+} \rightarrow-q_{1}^{+}, q_{3}^{+} \rightarrow-q_{3}^{+}$, the second fraction equals the first one, up to a minus sign. A similar argument holds for all the other terms of the first group in (51): one always finds pairs of two permutations in which the pole at $q_{i}^{\prime-}=0$ cancels. As to the second group, one first shows, in exactly the same way, that there are no single poles in any $q_{i}^{\prime-}$. Finally, by adding all six permutations one easily sees that also the regions $q_{i}^{\prime-}=0, q_{j}^{\prime-}=0$ do not lead to any singularity, i.e. we have no singularities from the denominators $1 / q_{i}^{\prime-} q_{j}^{\prime-}(i \neq j)$. Having shown that in the sum over all six permutations there are no infrared singularities, we are allowed to add, in 
all denominators of $1 / q_{i}^{\prime-}$ small imaginary parts as intermediate regulators: $1 /\left(q_{i}^{\prime-} \pm i \epsilon\right)$. The prescription has to be the same for all permutations, and the final answer, of course, will not depend upon which prescription we have chosen. We find it convenient to use the principal value prescription, i.e. in (47) we substitute

$$
\int \frac{d q_{i}^{\prime-}}{{q_{i}^{\prime}}^{-}} \rightarrow \mathcal{P} \int \frac{d q_{i}^{\prime-}}{q_{i}^{\prime-}}=\int \frac{d q_{i}^{\prime-}}{2}\left(\frac{1}{q_{i}^{\prime-}+i \epsilon}+\frac{1}{{q_{i}^{\prime-}-i \epsilon}^{-}}\right)
$$

for each denominator.

In the third part of our argument we perform, for each permutation separately, the integrations over $q_{1}^{\prime-}$ and $q_{3}^{\prime-}$ (at fixed values of $q_{1}^{+}$and $q_{3}^{+}$). Detailed expressions are presented in the appendix, and we only quote the most important results: denoting the four regions $\left(q_{1}^{+}>0, q_{3}^{+}>0\right),\left(q_{1}^{+}<0, q_{3}^{+}<0\right),\left(q_{1}^{+}>0, q_{3}^{+}<0\right)$, and $\left(q_{1}^{+}<0, q_{3}^{+}>0\right)$ by $>>,<<,><,<>$, reps., we find:

(i) only the equal sign regions, $<<$ and $>>$, contribute

(ii) apart from the overall factor $1 / 2$, the results are the same as obtained from taking residues of the poles coming from $D_{1}$ and $D_{3}$, i.e. by putting the lines $k_{1}$ and $k_{3}$ on-shell. The factor $1 / 2$ is removed by summing over the two regions $>>$ and $<<$ : in the following we restrict the longitudinal variables $q_{1}^{+}$and $q_{3}^{+}$to be positive.

This completes the derivation of the result stated at the beginning of this subsection: in Fig. 1 the upper and lower vertical $s$-channel gluons with momenta $k_{1}$ and $k_{3}$ are onshell, and the longitudinal ' $+{ }^{\prime}$ components of $k_{1}$ and $k_{3}$ must have equal sign. We could therefore use our expressions of the RPR and RPPR vertices, given in the appendix, with on-shell s-channel gluons, and combine them with the results of the integrations over the $q_{i}^{\prime-}$ components.

Nevertheless, we find it convenient to return to Fig.4, i.e. to the expressions for the kernel before our use of the Ward identities. The reason is that, in the integrals $I_{2}, I_{3}$, and $I_{4}$ and $I_{6}$ one finds the denominator $D_{13}$ which resulted from inserting the pole values for $q_{1}^{\prime-}$ and $q_{3}^{\prime-}$ from $D_{1}$ and $D_{3}$ into the denominator $-1 / q_{2}^{\prime-}=1 /\left(q_{1}^{\prime-}+q_{3}^{\prime-}\right)$. In the subsequent integration over the longitudinal $q_{i}^{+}$variables the presence of this denominator will lead to unwanted poles, e.g. of the form $1 /\left(k_{1 \perp}^{2}-k_{3 \perp}^{2}\right)$, which in the final result will cancel. In order to avoid this unnecessary complication it is more convenient to return to Fig.4. On the right hand side of Fig.4, only the first diagram has the two $s$-channel propagator poles in $D_{1}$ and $D_{3}$, and all the other diagrams can be disregarded.

\subsection{Final expression for the $3 \rightarrow 3$ vertex}

After this rather long 'detour' we address the final step and compute the first diagram on the right hand side of Fig.4, with on-shell $s$-channel gluons with momenta $k_{1}$ and $k_{3}$. Beginning with the RPPR vertex in the center (Fig.3e), it is convenient to make use of the existing two gluon production vertex in quasi-multi-Regge kinematics. This vertex has been calculated before, in the context of the NLO calculation of the BFKL kernel [26]. A convenient derivation starting from the effective action has been described in [14]. For our discussion we start from the expression given in [25]. Denoting the momenta of the two produced $s$-channel gluons by $k_{1}$ and $k_{3}$, and turning them into incoming momenta, 
we arrive at the tensor:

$$
\begin{aligned}
b^{\alpha \beta}\left(x_{1}\right)= & -\vec{q}_{2}^{2} \frac{k_{1}^{\alpha} k_{3}^{\beta}}{\vec{k}_{1}^{2} \vec{k}_{3}^{2}}- \\
& -\frac{g^{\alpha \beta}}{2} \frac{x_{1} x_{3}\left(\vec{q}_{2}^{2}+2 \vec{q}_{2} \vec{k}_{1}\right)}{\sigma_{1}}-\frac{x_{3} k_{1}^{\alpha} q_{2}^{\beta}+x_{1} q_{2}^{\alpha}\left(q_{2}+k_{1}\right)^{\beta}}{\sigma_{1}}-\frac{x_{1} \vec{q}_{2}^{2} k_{1}^{\alpha}\left(q_{2}+k_{1}\right)^{\beta}}{\vec{k}_{1}^{2} \sigma_{1}} \\
& -\frac{g^{\alpha \beta}}{2} \frac{x_{1} x_{3}\left(\vec{q}_{2}^{2}+2 \vec{q}_{2} \vec{k}_{3}\right)}{\sigma_{3}}-\frac{x_{1} q_{2}^{\alpha} k_{3}^{\beta}+x_{3}\left(q_{2}+k_{3}\right)^{\alpha} q_{2}^{\beta}}{\sigma_{3}}-\frac{x_{3} \vec{q}_{2}^{2} k_{3}^{\beta}\left(q_{2}+k_{3}\right)^{\alpha}}{\vec{k}_{3}^{2} \sigma_{3}},
\end{aligned}
$$

where the second line belongs to the first diagram in Fig.3e, the third line to the second diagram, and we have introduced $k_{i \perp}^{2}=-\vec{k}_{i}^{2}$. The denominators in (55) are defined as

$$
\begin{gathered}
\sigma_{1}=x_{1}\left(\vec{q}_{2}+\vec{k}_{1}\right)^{2}+x_{3} \vec{k}_{1}^{2} \\
\sigma_{3}=x_{3}\left(\vec{q}_{2}+\vec{k}_{3}\right)^{2}+x_{1} \vec{k}_{3}^{2} .
\end{gathered}
$$

We have rescaled our $q_{i}^{+}$variables (both $q_{1}^{+}$and $q_{2}^{+}$are positive):

$$
q_{i}^{+}=x_{i} q^{+}, i=1,3 ; q^{+}=q_{1}^{+}+q_{3}^{+} .
$$

The integral over $q^{+}$yields the logarithm of the energy corresponding to the BFKL evolution, whereas the integration over $x_{1}$ is a part of the RPPR vertex.

This expression has to be integrated over $x_{1}$ with the integration measure $1 /\left(x_{1}\left(1-x_{1}\right)\right)$. The integral diverges logarithmically at $x_{1}=1$ and at $x_{1}=0$. To be specific, let us consider Fig.5 (which corresponds to the first diagram in Fig.3e): at $x_{1}=0$ we have $q_{3}^{+} \gg q_{1}^{+}$, i.e. we are in the kinematical region in which the gluons with momenta $k_{1}$ and $k_{3}$ are separated by a large rapidity interval, and the gluon with momentum $k_{2}=k_{1}-q_{2}^{\prime}$ becomes a $t$-channel reggeized gluon having negligible longitudinal momenta. This configuration belongs to the leading order BKP evolution and, in order to find the next-to-leading order result, we have to subtract this contribution. A similar procedure holds for the limit $x_{1} \rightarrow 0$. For the NLO result we impose the intermediate rapidity cut-off:

$$
\left|y_{1}-y_{3}\right|<\Delta
$$

where $y_{i}=\ln \left(q_{i}^{+} /\left|\vec{k}_{i}\right|\right)(i=1,3)$ denotes the rapidity of gluon $i$. Including the subtraction of the LO contributions proportional to $b^{\alpha \beta}(0)+b^{\alpha \beta}(1)$ we find the following regularization prescription:

$$
\begin{array}{r}
\left.\int_{0}^{1} \frac{d x_{1}}{x_{1}\left(1-x_{1}\right)} b^{\alpha \beta}\left(x_{1}\right)\right|_{+}=\int_{e^{-\Delta} \frac{\left|\vec{k}_{1}\right|}{\left|\vec{k}_{3}\right|}}^{1-e^{-\Delta} \frac{\left|\vec{k}_{3}\right|}{\left|\vec{k}_{1}\right|}} \frac{d x_{1}}{x_{1}\left(1-x_{1}\right)} b^{\alpha \beta}\left(x_{1}\right)-\Delta\left(b^{\alpha \beta}(0)+b^{\alpha \beta}(1)\right) \\
=\int_{0}^{1} \frac{d x_{1}}{x_{1}}\left(b^{\alpha \beta}\left(x_{1}\right)-b^{\alpha \beta}(0)\right)+\int_{0}^{1} \frac{d x_{1}}{1-x_{1}}\left(b^{\alpha \beta}\left(x_{1}\right)-b^{\alpha \beta}(1)\right) \\
+b^{\alpha \beta}(0) \ln \frac{\left|\vec{k}_{3}\right|}{\left|\vec{k}_{1}\right|}+b^{\alpha \beta}(1) \ln \frac{\left|\vec{k}_{1}\right|}{\left|\vec{k}_{3}\right|}
\end{array}
$$


where, in the second line, we have taken the limit $\Delta \rightarrow \infty$. The integration over $x_{1}$ leads to the following expression for the RRPP vertex: .

$$
\begin{aligned}
V^{\alpha \beta}= & \ln \frac{\left(\vec{q}_{2}+\vec{k}_{1}\right)^{2}}{\sqrt{\vec{k}_{1}^{2} \vec{k}_{3}^{2}}}\left(-\frac{g^{\alpha \beta}}{2}+\frac{k_{1}^{\alpha} q_{2}^{\beta}}{\vec{k}_{1}^{2}}-\frac{\vec{q}_{2}^{2}}{\left(\vec{q}_{2}+\vec{k}_{1}\right)^{2}} \frac{k_{1}^{\alpha}\left(q_{2}+k_{1}\right)^{\beta}}{\vec{k}_{1}^{2}}-\frac{q_{2}^{\alpha}\left(q_{2}+k_{1}\right)^{\beta}}{\left(\vec{q}_{2}+\vec{k}_{1}\right)^{2}}\right)+ \\
& +\ln \frac{\left(\vec{q}_{2}+\vec{k}_{3}\right)^{2}}{\sqrt{\vec{k}_{1}^{2} \vec{k}_{3}^{2}}}\left(-\frac{g^{\alpha \beta}}{2}+\frac{q_{2}^{\alpha} k_{3}^{\beta}}{\vec{k}_{3}^{2}}-\frac{\vec{q}_{2}^{2}}{\left(\vec{q}_{2}+\vec{k}_{3}\right)^{2}} \frac{\left(q_{2}+k_{3}\right)^{\alpha} k_{3}^{\beta}}{\vec{k}_{3}^{2}}-\frac{\left(q_{2}+k_{3}\right)^{\alpha} q_{2}^{\beta}}{\left(\vec{q}_{2}+\vec{k}_{3}\right)^{2}}\right) .
\end{aligned}
$$

Including the contraction with the effective vertices from above and below we can write the result in the simplified form:

$$
\begin{aligned}
& K_{123}=\left(\frac{q_{1}^{\alpha}}{\vec{q}_{1}^{2}}-\frac{k_{1}^{\alpha}}{\vec{k}_{1}^{2}}\right) V_{\alpha \beta}\left(\frac{q_{3}^{\beta}}{\vec{q}_{3}^{2}}-\frac{k_{3}^{\beta}}{\vec{k}_{3}^{2}}\right) \\
&=-\left(\frac{q_{1}^{\alpha}}{\vec{q}_{1}^{2}}-\frac{k_{1}^{\alpha}}{\vec{k}_{1}^{2}}\right)\left[\ln \frac{\left(\vec{q}_{2}+\vec{k}_{1}\right)^{2}}{\sqrt{\vec{k}_{1}^{2} \vec{k}_{3}^{2}}} T_{\alpha \beta}\left(k_{1}\right) T_{\beta \gamma}\left(q_{2}+k_{1}\right)+\right. \\
&\left.+\ln \frac{\left(\vec{q}_{2}+\vec{k}_{3}\right)^{2}}{\sqrt{\vec{k}_{1}^{2} \vec{k}_{3}^{2}}} T_{\alpha \beta}\left(q_{2}+k_{3}\right) T_{\beta \gamma}\left(k_{3}\right)\right]\left(\frac{q_{3}^{\gamma}}{\vec{q}_{3}^{2}}-\frac{k_{3}^{\gamma}}{\vec{k}_{3}^{2}}\right),
\end{aligned}
$$

where

$$
T_{\alpha \beta}(k)=g_{\alpha \beta}-2 \frac{k_{\alpha} k_{\beta}}{\vec{k}^{2}} .
$$

In (61) we have included the propagators for the two gluons with momenta $q_{1}$ and $q_{3}$. For later convenience it is useful to present the vertex also in complex notation. Introducing $q=q_{x}+i q_{y}, q^{*}=q_{x}-i q_{y}$ etc. we find:

$$
\begin{array}{r}
\tilde{K}_{123}=-\frac{1}{4} \frac{1}{q_{2} q_{2}^{*}}\left\{\log \frac{\left|q_{2}+k_{1}\right|^{2}}{\left|k_{1}\right|\left|k_{3}\right|}\left[\frac{q_{1}^{\prime *} q_{3}^{\prime}}{q_{1}^{*} q_{3}} \frac{1}{k_{1} k_{3}} \frac{\left(q_{2}+k_{1}\right)}{\left(q_{2}+k_{1}\right)^{*}}+\text { c.c. }\right]+\right. \\
\left.\log \frac{\left|q_{2}+k_{3}\right|^{2}}{\left|k_{1}\right|\left|k_{3}\right|}\left[\frac{q_{1}^{\prime} q_{3}^{\prime *}}{q_{1} q_{3}^{*}} \frac{1}{k_{1} k_{3}} \frac{\left(q_{2}+k_{3}\right)}{\left(q_{2}+k_{3}\right)^{*}}+\text { c.c. }\right]\right\} .
\end{array}
$$

Here we have included also the propagator of the gluon with momentum $q_{2}$.

Let us mention a few important properties of this $3 \rightarrow 3$ vertex. First we note that the poles from the propagators of the $s$-channel gluon with momentum $k_{2}$ in (60) cancel: this is seen most directly in the complex representation (63). Next we note that the vertex, after removing the propagators on the left side of the $3 \rightarrow 3$ vertex, vanishes as any of the external momenta $\vec{q}_{i}, \vec{q}_{i}^{\prime}$ goes to zero, in agreement with the arguments of section 3.1 based on the Ward identities.

This completes our derivation of the $3 \rightarrow 3$ vertex. Together with the NLO $2 \rightarrow 2$ kernel presented in section 2, it constitutes the NLO kernel of the BKP evolution equation. 


\section{Conclusions}

In this paper we have presented analytic expressions of the NLO kernel of the BKP evolution equations in the Odderon channel, consisting of the NLO corrections of the $2 \rightarrow 2$ BFKL kernel in the symmetric color octet channel and of the Born approximation of the connected $3 \rightarrow 3$ transition kernel. In deriving the final form of the $3 \rightarrow 3$ (eqs.(61) and (63)) we found it helpful to make use of Ward identities of amplitudes of reggeized gluons which have been derived recently. In [15] these Ward identities had been used for obtaining a new derivation of the BFKL kernel; in the present paper we have derived, by the same methods, compact expressions for the RPRR, RRPR, and RPPR vertices. Starting from the results of the $2 \rightarrow 2$ and $3 \rightarrow 3$ transition kernels it will be possible to calculate the NLO intercept of the Odderon solution. Work along these lines is in progress.

Our NLO calculation of the $2 \rightarrow 2$ kernel also applies to the large- $N_{c}$ limit of the Ceven and odd BKP equations of $n \geq 3$ reggeized gluons in the color singlet state. In the (planar) large- $N_{c}$ limit any pair of neighboring reggeized gluons is in an octet state, and the gluon trajectories of the $n$ gluon lines can be distributed among the $2 \rightarrow 2$ kernels in such way that the kernels are infrared finite. Our results can also be used for the large $N_{c}$ limit of the BKP-equations for n reggeized gluons in the adjoint representation. This case has attracted interest in the context of studies of the AdS/CFT duality, in particular for the computation of the remainder function. Compared to the color singlet case, the infrared finiteness of the $2 \rightarrow 2$ kernels now works in a slightly different way. Labeling the reggeized gluons of the n-gluon state by $1, \ldots, n$ with momenta $q_{1}, \ldots q_{n}$, we distribute the gluon trajectories in the same way as in the singlet case. But since in the planar approximation of the adjoint representation, when compared to the color singlet configuration which lives on the surface of a cylinder, one $2 \rightarrow 2$ kernel is missing (namely the interaction between reggeons $n$ and 1), the compensation of infrared singularities is incomplete. In order to achieve infrared finiteness, we subtract the gluon trajectory as a function the sum of all transverse momenta, $q=q_{1}+\ldots+q_{n}$. This subtraction is in agreement with the fact that, in the BDS formula, this trajectory is contained already in the BDS part and thus should not be part of the remainder function. In this way, also the color octet case is infrared finite, and should have the dual conformal invariance in momentum space, observed in [7] and [10].

Our derivation of the $3 \rightarrow 3$ transition kernel, so far, is restricted to the system of three reggeized gluons in the Odderon channel. Its use for the C-even odd-signature three gluon contribution to the spin structure function $g_{2}$ [27], as well as for the large- $N_{c}$ limit of the C-even and odd BKP equations of $n$ reggeized gluons in the color singlet state requires a few additional steps which will be presented in a future paper; the same applies also to its use for the remainder function of the $2 \rightarrow n$ amplitude within the AdS/CFT duality. In a future paper we hope to come back to these generalizations.

Acknowledements: This work has been supported by the SFB 676 'Particles, Strings and the Early Universe: the structure of Matter and Space-Time'. Three of us (V.S.Fadin, L..Lipatov, and G.P.Vacca) express their gratitude for the hospitality of DESY and of the 
II.Institut of Theoretical Physics, Hamburg University. One of us (J.Bartels) thanks the INFN and the University of Bologna for their hospitality.

\section{A Appendix}

In this appendix we derive the numerator of the sum of diagrams shown in Fig.5. Let us first describe the building blocks. Beginning with the left hand part of Fig.5a, the production vertices at the upper and at the lower end (black boxes) are obtained from the effective production vertices illustrated in the first line of Fig.3 by removing the induced contributions. What is left are the QCD triple gluon vertices, contracted with transverse momenta from the left and from the right hand side (see eq.(46)). Below the upper production vertex we have the left central vertex and the right central vertex: they are obtained from the effective vertices in the second line of Fig.3 by disregarding the induced vertices and by multiplying with the corresponding transverse momenta: this leads to the QCD triple gluon vertices with a transverse momentum contraction for the reggeized gluon. At the end, we have to re-add the induced terms for the gluons with momenta $q_{2}$ and $q_{2}^{\prime}$.

\section{A.1 Building blocks}

We collect the building blocks. The overall multiplicative factor is

$$
\frac{1}{D_{1} D_{2} D_{3}} \frac{2^{6}}{q_{1}^{+} q_{2}^{+} q_{3}^{+} q_{1}^{\prime-} q_{2}^{\prime-} q_{3}^{\prime-}}
$$

with

$$
\begin{aligned}
& D_{1}=k_{1}^{2}+i \epsilon=q_{1}{ }^{+}\left(-q_{1}^{\prime}\right)^{-}+k_{1 \perp}^{2}+i \epsilon \\
& D_{2}=k_{2}^{2}+i \epsilon=\quad q_{1}^{+} q_{3}^{\prime-}+k_{2 \perp}^{2}+i \epsilon \\
& D_{3}=k_{3}^{2}+i \epsilon=q_{3}^{+}\left(-q_{3}^{\prime}\right)^{-}+k_{3 \perp}^{2}+i \epsilon .
\end{aligned}
$$

Next we list the four vertices (black boxes) in the left column of Fig.5a. For the QCD triple gluon vertex

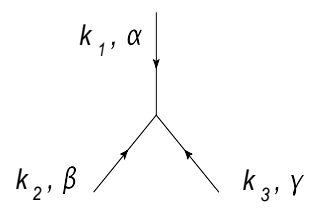

we use the notation

$$
\gamma^{\alpha \beta \gamma}\left(k_{1},-k_{3}\right)=g^{\alpha \beta}\left(k_{1}-k_{2}\right)^{\gamma}+g^{\beta \gamma}\left(k_{2}-k_{3}\right)^{\alpha}+g^{\gamma \alpha}\left(k_{3}-k_{1}\right)^{\beta} .
$$

For the first term, the upper vertex, we have from [15]:

$$
\begin{array}{r}
\left(\left(q_{1}\right)_{\perp \lambda} \gamma^{\lambda \alpha \lambda^{\prime}}\left(q_{1}, q_{1}^{\prime}\right)\left(q_{1}^{\prime}\right)_{\perp \lambda^{\prime}}\right)= \\
\left(q_{1 \perp}\right)^{\alpha}\left(q_{1}-q_{1}^{\prime}\right)_{\perp} q_{1 \perp}^{\prime}-\left(q_{1 \perp}^{\prime}\right)^{\alpha}\left(q_{1}-q_{1}^{\prime}\right)_{\perp} q_{1 \perp}-\left(\frac{\left(n^{+}\right)^{\alpha}}{2}\left(q_{1}^{\prime}\right)^{-}+\frac{\left(n^{-}\right)^{\alpha}}{2}\left(q_{1}\right)^{+}\right) q_{1 \perp} q_{1 \perp}^{\prime} .
\end{array}
$$


For the left central vertex for the gluon with momentum $q_{2}^{\prime}$ we find:

$$
\begin{gathered}
\gamma^{\beta \alpha \mu^{\prime}}\left(-k_{2}, q_{2}^{\prime}\right)\left(q_{2 \perp}^{\prime}\right)_{\mu^{\prime}}=-g^{\alpha \beta} q_{2 \perp}^{\prime}\left(k_{2}+k_{1}\right)_{\perp} \\
+\frac{\left(n^{+}\right)^{\beta}}{2}\left(q_{2 \perp}^{\prime}\right)^{\alpha}\left(-q_{1}^{\prime}+q_{2}^{\prime}\right)^{-}+\frac{\left(n^{-}\right)^{\beta}}{2}\left(q_{2 \perp}^{\prime}\right)^{\alpha} q_{1}^{+}+\frac{\left(n^{+}\right)^{\alpha}}{2}\left(q_{2 \perp}^{\prime}\right)^{\beta}\left(-q_{2}^{\prime}+q_{3}^{\prime}\right)^{-}+\frac{\left(n^{-}\right)^{\alpha}}{2}\left(q_{2 \perp}^{\prime}\right)^{\beta} q_{1}^{+} \\
+\left(q_{2 \perp}^{\prime}\right)^{\alpha}\left(k_{1}+q_{2}^{\prime}\right)_{\perp}^{\beta}+\left(q_{2 \perp}^{\prime}\right)^{\beta}\left(-q_{2}^{\prime}+k_{2}\right)_{\perp}^{\alpha},
\end{gathered}
$$

which we can also write in the form:

$$
\begin{array}{r}
\gamma^{\beta \alpha \mu^{\prime}}\left(-k_{2}, q_{2}^{\prime}\right)\left(q_{2 \perp}^{\prime}\right)_{\mu^{\prime}}= \\
-g^{\alpha \beta} q_{2 \perp}^{\prime}\left(k_{2}+k_{1}\right)_{\perp}+\left(q_{2 \perp}^{\prime}\right)^{\beta} k_{1}^{\alpha}+k_{2}^{\beta}\left(q_{2 \perp}^{\prime}\right)^{\alpha}-q_{2}^{\prime-}\left(\left(q_{2 \perp}^{\prime}\right)^{\beta}\left(n^{+}\right)^{\alpha}-\left(n^{+}\right)^{\beta}\left(q_{2 \perp}^{\prime}\right)^{\alpha}\right) .
\end{array}
$$

For the induced term we have:

$$
-\frac{q_{2}^{\prime-}}{2} \frac{q_{2 \perp}^{\prime 2}}{k_{1}^{+}}\left(n^{+}\right)^{\alpha}\left(n^{+}\right)^{\beta}=-\frac{q_{2 \perp}^{\prime 2}}{2} \frac{q_{2}^{\prime-}}{q_{1}^{+}}\left(n^{+}\right)^{\alpha}\left(n^{+}\right)^{\beta} .
$$

Similarly, the right central vertex for the gluon with momentum $q_{2}$ reads:

$$
\begin{gathered}
\left(q_{2 \perp}\right)_{\mu} \gamma^{\mu \beta \gamma}\left(q_{2},-k_{3}\right)=g^{\beta \gamma} q_{2 \perp}\left(k_{2}-k_{3}\right)_{\perp} \\
+\frac{\left(n^{+}\right)^{\gamma}}{2}\left(q_{2 \perp}\right)^{\beta}\left(-q_{3}^{\prime}\right)^{-}+\frac{\left(n^{-}\right)^{\gamma}}{2}\left(q_{2 \perp}\right)^{\beta}\left(q_{2}-q_{1}\right)^{+}+\frac{\left(n^{+}\right)^{\beta}}{2}\left(q_{2 \perp}\right)^{\gamma}\left(-q_{3}^{\prime}\right)^{-}+\frac{\left(n^{-}\right)^{\beta}}{2}\left(q_{2 \perp}\right)^{\gamma}\left(q_{3}-q_{2}\right)^{+} \\
+\left(q_{2 \perp}\right)^{\beta}\left(q_{2}-k_{2}\right)_{\perp}^{\gamma}+\left(q_{2 \perp}\right)^{\gamma}\left(k_{3}-q_{2}\right)_{\perp}^{\beta},
\end{gathered}
$$

for which we also have:

$$
\begin{array}{r}
\left(q_{2 \perp}\right)_{\mu} \gamma^{\mu \beta \gamma}\left(q_{2},-k_{3}\right) \\
g^{\beta \gamma} q_{2 \perp}\left(k_{2}-k_{3}\right)_{\perp}+\left(q_{2 \perp}\right)^{\beta} k_{3}^{\gamma}-k_{2}^{\beta}\left(q_{2 \perp}\right)^{\gamma}+q_{2}^{+}\left(\left(q_{2 \perp}\right)^{\beta}\left(n^{-}\right)^{\gamma}-\left(n^{-}\right)^{\beta}\left(q_{2 \perp}\right)^{\gamma}\right) .
\end{array}
$$

The induced term for this reggeized gluon is:

$$
-\frac{\left(q_{2}\right)^{+}}{2} \frac{q_{2 \perp}^{2}}{k_{3}^{-}}\left(n^{-}\right)^{\beta}\left(n^{-}\right)^{\gamma}=\frac{q_{2 \perp}^{2}}{2} \frac{\left(q_{2}\right)^{+}}{\left(q_{3}^{\prime}\right)^{-}}\left(n^{-}\right)^{\beta}\left(n^{-}\right)^{\gamma} .
$$

Finally the lower vertex:

$$
\begin{array}{r}
\left(\left(q_{3}\right)_{\perp \nu} \gamma^{\nu \gamma \nu^{\prime}}\left(q_{3},-q_{3}^{\prime}\right)\left(q_{3}^{\prime}\right)_{\perp \nu^{\prime}}\right)= \\
\left(q_{3 \perp}\right)^{\gamma}\left(q_{3}-q_{3}^{\prime}\right)_{\perp} q_{3 \perp}^{\prime}-\left(q_{3 \perp}^{\prime}\right)^{\gamma}\left(q_{3}-q_{3}^{\prime}\right)_{\perp} q_{3 \perp}-\left(\frac{\left(n^{+}\right)^{\gamma}}{2}\left(q_{3}^{\prime}\right)^{-}+\frac{\left(n^{-}\right)^{\gamma}}{2}\left(q_{3}\right)^{+}\right) q_{3 \perp} q_{3 \perp}^{\prime} .
\end{array}
$$

For the upper line in Fig.5a, the contribution of the quartic coupling is:

$$
\left(q_{1 \perp}\right)^{\beta} q_{2 \perp}^{\prime} q_{1 \perp}^{\prime}-\left(q_{1 \perp}^{\prime}\right)^{\beta} q_{2 \perp}^{\prime} q_{1 \perp} .
$$

Similarly for the lower line:

$$
\left(q_{3 \perp}\right)^{\beta} q_{2 \perp} q_{3 \perp}^{\prime}-\left(q_{3 \perp}^{\prime}\right)^{\beta} q_{2 \perp} q_{3 \perp} .
$$

Finally, the central part of Fig.5b is given by:

$$
g^{\alpha \gamma} q_{2 \perp} q_{2 \perp}^{\prime}-\left(q_{2 \perp}\right)^{\alpha}\left(q_{2 \perp}^{\prime}\right)^{\gamma} .
$$




\section{A.2 The effective RPRR and RRPR vertices}

Next we write down the explicit expression for the lower RRPR vertex (lower line in Fig.5a). Combining (A.11), (A.13), (A.12), and (A.15) we obtain:

$$
\begin{gathered}
-q_{2}^{+} q_{3}^{\prime-} q_{2 \perp}^{\beta} q_{3 \perp} q_{3 \perp}^{\prime}-k_{2}^{\beta}\left(q_{2 \perp} \times k_{3 \perp}\right)\left(q_{3 \perp} \times q_{3 \perp}^{\prime}\right)+\left(k_{3}^{+} k_{3}^{-}+k_{3 \perp}^{2}\right)\left(n_{\perp}^{\beta} \times q_{2 \perp}\right)\left(q_{3 \perp} \times q_{3 \perp}^{\prime}\right) \\
-\left(\frac{\left(n^{+}\right)^{\beta}}{2}\left(q_{3}^{\prime}\right)^{-}+\frac{\left(n^{-}\right)^{\beta}}{2}\left(q_{3}\right)^{+}\right) q_{3 \perp} q_{3 \perp}^{\prime} q_{2 \perp}\left(k_{2 \perp}-k_{3 \perp}\right)-\left(q_{2}\right)^{+}\left(n^{-}\right)^{\beta}\left(q_{2 \perp} \times k_{3 \perp}\right)\left(q_{3 \perp} \times q_{3 \perp}^{\prime}\right) \\
\quad+\left(n_{\perp}^{\beta} \times k_{3 \perp}\right)\left(q_{3 \perp} \times q_{3 \perp}^{\prime}\right) q_{2 \perp}\left(k_{2 \perp}-k_{3 \perp}\right)-\frac{\left(n^{-}\right)^{\beta}}{2}\left(q_{2}\right)^{+} q_{2 \perp}^{2} q_{3 \perp} q_{3 \perp}^{\prime},
\end{gathered}
$$

where we have introduced the vector product-like notation $(a \times b)=a_{x} b_{y}-a_{y} b_{x}$ and defined the unit vector $n_{\perp}^{\beta}$ with $n_{\perp}^{\beta} q_{\perp}=\left(q_{\perp}\right)^{\beta}$. Alternatively we can write:

$$
\begin{array}{r}
\frac{\left(n^{-}\right)^{\beta}}{2}\left[-\left(q_{3}\right)^{+} q_{3 \perp} q_{3 \perp}^{\prime} q_{2 \perp}\left(k_{2 \perp}-k_{3 \perp}\right)+\left(q_{3}-q_{2}\right)^{+}\left(q_{2 \perp} \times k_{3 \perp}\right)\left(q_{3 \perp} \times q_{3 \perp}^{\prime}\right)-\left(q_{2}\right)^{+} q_{2}^{2} q_{3 \perp} q_{3 \perp}^{\prime}\right] \\
\quad+\frac{\left(n^{+}\right)^{\beta}}{2}\left[-\left(q_{3}^{\prime}\right)^{-} q_{3 \perp} q_{3 \perp}^{\prime} q_{2 \perp}\left(k_{2 \perp}-k_{3 \perp}\right)-\left(q_{3}^{\prime}\right)^{-}\left(q_{2 \perp} \times k_{3 \perp}\right)\left(q_{3 \perp} \times q_{3 \perp}^{\prime}\right)\right] \\
\quad-q_{2}^{+} q_{3}^{\prime-} q_{2 \perp}^{\beta} q_{3 \perp} q_{3 \perp}^{\prime}+\left(-q_{3}^{+} q_{3}^{\prime-}+k_{3 \perp}^{2}\right)\left(n_{\perp}^{\beta} \times q_{2 \perp}\right)\left(q_{3 \perp} \times q_{3 \perp}^{\prime}\right) \\
-k_{2 \perp}^{\beta}\left(q_{2 \perp} \times k_{3 \perp}\right)\left(q_{3 \perp} \times q_{3 \perp}^{\prime}\right)+\left(n_{\perp}^{\beta} \times k_{3 \perp}\right)\left(q_{3 \perp} \times q_{3 \perp}^{\prime}\right) q_{2 \perp}\left(k_{2 \perp}-k_{3 \perp}\right) .
\end{array}
$$

One verifies that (A.18) satisfies the Ward identity, i.e. after contracting with $\left(k_{2}\right)_{\beta}$ we obtain zero. We also note that if we put the $k_{2}$-line on mass shell and multiply with a physical (transverse) polarization vector, the induced term of the reggeized gluon with momentum $q_{2}$ does not contribute.

An analogous expression is obtained for the upper RPRR vertex (upper line in Fig.5a):

$$
\begin{array}{r}
\frac{\left(n^{+}\right)^{\beta}}{2}\left[\left(q_{1}^{\prime}\right)^{-} q_{1 \perp} q_{1 \perp}^{\prime} q_{2 \perp}^{\prime}\left(k_{1 \perp}+k_{2 \perp}\right)+\left(q_{2}^{\prime}-q_{1}^{\prime}\right)^{-}\left(q_{2 \perp}^{\prime} \times k_{1 \perp}\right)\left(q_{1 \perp} \times q_{1 \perp}^{\prime}\right)+\left(q_{2}^{\prime}\right)^{-} q_{2 \perp}^{\prime}{ }^{2} q_{1 \perp} q_{1 \perp}^{\prime}\right] \\
+\frac{\left(n^{-}\right)^{\beta}}{2}\left[\left(q_{1}\right)^{+} q_{1 \perp} q_{1 \perp}^{\prime} q_{2 \perp}^{\prime}\left(k_{1 \perp}+k_{2 \perp}\right)+\left(q_{1}\right)^{+}\left(q_{2 \perp}^{\prime} \times k_{1 \perp}\right)\left(q_{1 \perp} \times q_{1 \perp}^{\prime}\right)\right] \\
\quad+q_{2}^{\prime-} q_{1}^{+}\left(q_{2 \perp}^{\prime}\right)^{\beta} q_{1 \perp} q_{1 \perp}^{\prime}+\left(-q_{1}^{+} q_{1}^{\prime-}+k_{1 \perp}^{2}\right)\left(n_{\perp}^{\beta} \times q_{2 \perp}^{\prime}\right)\left(q_{1 \perp} \times q_{1 \perp}^{\prime}\right) \\
+k_{2 \perp}^{\beta}\left(q_{2 \perp}^{\prime} \times k_{1 \perp}\right)\left(q_{1 \perp} \times q_{1 \perp}^{\prime}\right)-\left(n_{\perp}^{\beta} \times k_{1 \perp}\right)\left(q_{1 \perp} \times q_{1 \perp}^{\prime}\right) q_{2 \perp}^{\prime}\left(k_{1 \perp}+k_{2 \perp}\right) .
\end{array}
$$

The contribution of the quartic coupling in Fig.5b reads:

$$
\begin{array}{r}
\left(q_{1}^{+} q_{3}^{\prime-}+k_{2 \perp}^{2}\right)\left[\frac{1}{2}\left(q_{3}^{+} q_{1}^{\prime-}+q_{1}^{+} q_{3}^{\prime-}\right) q_{1 \perp} q_{1 \perp}^{\prime} q_{2 \perp} q_{2 \perp}^{\prime}, q_{3 \perp} q_{3 \perp}^{\prime}+\right. \\
\left.k_{1 \perp} q_{2 \perp}^{\prime} k_{3 \perp} q_{2 \perp}\left(q_{1 \perp} \times q_{1 \perp}^{\prime}\right)\left(q_{3 \perp} \times q_{3 \perp}^{\prime}\right)\right] .
\end{array}
$$

For later purposes we remind that the production vertex in (A.6) satisfies the Ward identity, i.e. it vanishes when contracted with $k_{1}$. The same applies to the lower production vertex (A.13) and its contraction with $k_{3}$, resp.. 


\section{A.3 The effective RPPR vertex}

Furthermore, from the building blocks listed in subsection A.1 we can form the gauge invariant RPPR vertex (Fig.6). To this end we combine, in Fig.6a, the left and right central vertices, (A.8) and (A.11) (together with their induced terms). In Fig.6b we collect the mirror reflected diagrams of Fig.6a. Fig.6c shows the nonplanar diagram (which in the odderon case drops out, once we convolute with the impact factors), and Fig.6d shows the quartic coupling (A.16).

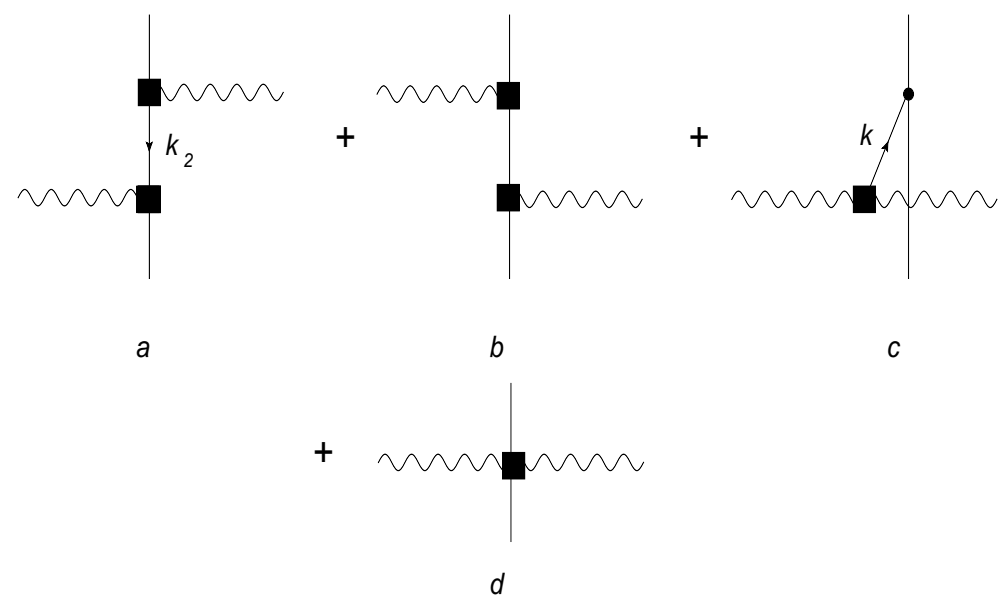

Fig.6: The effective RPPR vertex

The sum of these four structures $(a)+(b)+(c)+(d)$ is gauge invariant, i.e it satisfies the Ward identity in $k_{1}$, provided the gluon with momentum $k_{3}$ is on shell and we multiply with a physical polarization vector. Decomposing the three different color tensors in the two independent ones, making use of the Jacobi identity on the non planar contribution, we can verify that the corresponding combination of each planar structure with the non planar one separately satisfies the Ward identity. Let us define the color stripped contribution corresponding to Fig.6a as

$$
\begin{aligned}
& I_{a}^{\alpha \gamma}\left(k_{1}, k_{3}\right)=g^{\alpha \gamma} q_{2 \perp} q_{2 \perp}^{\prime}-\left(q_{2 \perp}\right)^{\alpha}\left(q_{2 \perp}^{\prime}\right)^{\gamma}+ \\
& \quad\left[\gamma^{\beta \alpha \mu^{\prime}}\left(-k_{2}, q_{2}^{\prime}\right)\left(q_{2 \perp}^{\prime}\right)_{\mu^{\prime}}-\frac{q_{2}^{\prime}}{2} \frac{q_{2 \perp}^{\prime 2}}{k_{1}^{+}}\left(n^{+}\right)^{\alpha}\left(n^{+}\right)^{\beta}\right] \frac{1}{k_{2}^{2}}\left[\left(q_{2 \perp}\right)_{\mu} \gamma^{\mu \beta \gamma}\left(q_{2},-k_{3}\right)-\frac{\left(q_{2}\right)^{+}}{2} \frac{q_{2 \perp}^{2}}{k_{3}^{-}}\left(n^{-}\right)^{\beta}\left(n^{-}\right)^{\gamma}\right] .
\end{aligned}
$$

Here the first line contains the contribution of the quartic coupling, and in the second line we have put $k_{2}=k_{1}-q_{2}^{\prime}=-k_{3}-q_{2}$. The nonplanar contribution corresponding to Fig.6c can be written as

$$
\begin{aligned}
& I_{c}^{\alpha \gamma}\left(k_{1}, k_{3}\right)=q_{2 \perp}^{\alpha}\left(q_{2 \perp}^{\prime}\right)^{\gamma}-\left(q_{2 \perp}\right)^{\gamma}\left(q_{2 \perp}^{\prime}\right)^{\alpha}+ \\
& \quad\left(\left(q_{2}\right)_{\perp \mu} \gamma^{\mu \beta \mu^{\prime}}\left(q_{2}, q_{2}^{\prime}\right)\left(q_{2}^{\prime}\right)_{\perp \mu^{\prime}}\right) \frac{1}{k^{2}} \gamma^{\alpha \beta \gamma}\left(k_{1},-k_{3}\right) .
\end{aligned}
$$

Again, the first line contains the quartic coupling term, and in the second line we have used $k=q_{2}-q_{2}^{\prime}=-k_{1}-k_{3}$.

In terms of these quantities the Ward identity we want to check is given by

$$
k_{1 \alpha}\left(I_{a}^{\alpha \gamma}-I_{c}^{\alpha \gamma}\right) \epsilon_{3 \gamma}=0
$$


where $\epsilon_{3 \gamma} k_{3}^{\gamma}=0$ and $k_{3}^{2}=0$. In order to show it one can use the following identities:

$$
\begin{aligned}
& k_{1 \alpha}\left[\gamma\left(-k_{2}, q_{2}^{\prime}\right)^{\beta \alpha \mu^{\prime}}\left(q_{2 \perp}^{\prime}\right)_{\mu^{\prime}}-\frac{q_{2}^{\prime}}{2} \frac{q_{2 \perp}^{\prime 2}}{k_{1}^{+}}\left(n^{+}\right)^{\alpha}\left(n^{+}\right)^{\beta}\right]=-k_{2}^{\beta} q_{2 \perp}^{\prime} k_{2 \perp}+\left(q_{2 \perp}^{\prime}\right)^{\beta} k_{2}^{2}, \\
& k_{2 \beta}\left[\left(q_{2 \perp}\right)_{\mu} \gamma\left(q_{2},-k_{3}\right)^{\mu \beta \gamma}-\frac{\left(q_{2}\right)^{+}}{2} \frac{q_{2 \perp}^{2}}{k_{3}^{-}}\left(n^{-}\right)^{\beta}\left(n^{-}\right)^{\gamma}\right]=k_{3}^{\gamma} q_{2 \perp} k_{3 \perp}-\left(q_{2 \perp}\right)^{\gamma} k_{3}^{2}, \\
& k_{1 \alpha}\left[g^{\alpha \gamma}\left(2 k_{3}+k\right)^{\beta}-g^{\alpha \beta}\left(k_{3}+2 k\right)^{\gamma}-g^{\beta \gamma}\left(k_{3}-k\right)^{\alpha}\right]=k^{\beta}\left(k+k_{3}\right)^{\gamma}-\left(k+k_{3}\right)^{\beta} k_{3}^{\gamma}+\left(k_{3}^{2}-k^{2}\right) g^{\beta \gamma},
\end{aligned}
$$

where in the first two lines (planar structure) one uses $k_{2}=k_{1}-q_{2}^{\prime}$, and in the last line (non planar structure) $k=-k_{1}-k_{3}$. Then one obtains

$$
\begin{aligned}
& k_{1 \alpha} \epsilon_{3 \gamma} I_{a}^{\alpha \gamma}=\epsilon_{3 \gamma} {\left[q_{2 \perp}^{\prime} k_{3 \perp}\left(q_{2 \perp}\right)^{\gamma}-q_{2 \perp} k_{3 \perp}\left(q_{2 \perp}^{\prime}\right)^{\gamma}+\frac{1}{2} q_{2 \perp} q_{2 \perp}^{\prime}\left[q_{2}^{+}\left(n^{-}\right)^{\gamma}+q_{2}^{\prime-}\left(n^{+}\right)^{\gamma}\right]\right], } \\
& k_{1 \alpha} \epsilon_{3 \gamma} I_{c}^{\alpha \gamma}=\epsilon_{3 \gamma}\left[\left(q_{2}-q_{2}^{\prime}\right)_{\perp} q_{2 \perp}\left(q_{2 \perp}^{\prime}\right)^{\gamma}-\left(q_{2}-q_{2}^{\prime}\right)_{\perp} q_{2 \perp}^{\prime}\left(q_{2 \perp}\right)^{\gamma}+\frac{1}{2} q_{2 \perp} q_{2 \perp}^{\prime}\left[q_{2}^{+}\left(n^{-}\right)^{\gamma}+q_{2}^{\prime}-\left(n^{+}\right)^{\gamma}\right]\right. \\
&\left.+q_{2 \perp} k_{1 \perp}\left(q_{2 \perp}^{\prime}\right)^{\gamma}-q_{2 \perp}^{\prime} k_{1 \perp}\left(q_{2 \perp}\right)^{\gamma}\right],
\end{aligned}
$$

which leads to (A.23).

Similarly one can write the color stripped contribution of Fig.6b

$$
\begin{aligned}
& I_{b}^{\alpha \gamma}\left(k_{1}, k_{3}\right)=\left(q_{2 \perp}^{\prime}\right)^{\alpha}\left(q_{2 \perp}\right)^{\gamma}-g^{\alpha \gamma} q_{2 \perp} q_{2 \perp}^{\prime}+ \\
& \quad\left[\left(q_{2 \perp}\right)_{\mu} \gamma^{\mu \alpha \beta}\left(q_{2}, k_{2}\right)-\frac{\left(q_{2}\right)^{+}}{2} \frac{q_{2 \perp}^{2}}{k_{1}^{-}}\left(n^{-}\right)^{\alpha}\left(n^{-}\right)^{\beta}\right] \frac{1}{k_{2}^{2}}\left[\gamma^{\gamma \beta \mu^{\prime}}\left(k_{3}, q_{2}^{\prime}\right)\left(q_{2 \perp}^{\prime}\right)_{\mu^{\prime}}-+\frac{q_{2}^{\prime-}}{2} \frac{q_{2 \perp}^{\prime 2}}{k_{3}^{+}}\left(n^{+}\right)^{\beta}\left(n^{+}\right)^{\gamma}\right],
\end{aligned}
$$

where $k_{2}=k_{1}+q_{2}=q_{2}^{\prime}-k_{3}$. Combined with the nonplanar contribution Fig.6c, one easily verifies the Ward identity.

In the context of this paper, in which we study the NLO corrections to the BKP equation for the Odderon channel (and for the large- $N_{c}$ limit of the multi-reggeon channel), the nonplanar structure of Fig.6c does not contribute, and the RPPR vertex reduces to $I_{a}^{\alpha \gamma}\left(k_{1}, k_{3}\right)+I_{b}^{\alpha \gamma}\left(k_{1}, k_{3}\right)$, written in eqs. (A.21) and (A.25). Now the Ward identities follow from adding Figs.6a and b.

\section{A.4 Putting things together}

Let us now combine these building blocks and obtain the numerator: $N=\mathrm{RRPR} \otimes \mathrm{RPRR}$ + quartic coupling. The prefactors are given in (A.1). The numerator is proportional to $q_{1 \perp}, q_{1 \perp}^{\prime}, q_{2 \perp}, q_{2 \perp}^{\prime}, q_{3 \perp}, q_{3 \perp}^{\prime}$. The dependence upon $q_{1 \perp}, q_{1 \perp}^{\prime}$ is through $q_{1 \perp} \cdot q_{1 \perp}^{\prime}$ or $q_{1 \perp} \times q_{1 \perp}^{\prime}$, similarly for $q_{3 \perp}$ and $q_{3 \perp}^{\prime}$. It is therefore convenient to organize the numerator in the following four groups:

1) terms proportional to $\left(q_{1 \perp} \times q_{1 \perp}^{\prime}\right)\left(q_{3 \perp} \times q_{3 \perp}^{\prime}\right)$ are:

$$
\begin{array}{r}
q_{1}^{+} q_{3}^{+} q_{1}^{\prime-} q_{3}^{\prime-} q_{2 \perp} q_{2 \perp}^{\prime} \\
-q_{1}^{+} q_{1}^{\prime-} q_{2 \perp}^{\prime} k_{2 \perp} q_{2 \perp} k_{3 \perp}+ \\
q_{1}^{+} q_{3}^{\prime-} q_{2 \perp}^{\prime} k_{1 \perp} q_{2 \perp} k_{3 \perp}+q_{3}^{+} q_{3}^{\prime-} q_{2 \perp} k_{2 \perp} q_{2 \perp}^{\prime} k_{1 \perp} \\
-\left(q_{2}^{+} q_{2}^{\prime-}+q_{3}^{+} q_{1}^{\prime-}\right)\left(q_{2 \perp} \times k_{3 \perp}\right)\left(q_{2 \perp}^{\prime} \times k_{1 \perp}\right) .
\end{array}
$$


Note that the sum of all terms without longitudinal momenta cancel.

2) terms proportional to $\left(q_{1 \perp} \cdot q_{1 \perp}^{\prime}\right)\left(q_{3 \perp} \times q_{3 \perp}^{\prime}\right)$ are:

$$
\begin{aligned}
q_{1}^{+} q_{2}^{\prime-}\left(q_{3}^{+}\right. & \left.q_{3}^{\prime-}\left(q_{2 \perp} \times q_{2 \perp}^{\prime}\right)-q_{2 \perp}^{\prime} k_{2 \perp}\left(q_{2 \perp} \times k_{3 \perp}\right)-k_{3 \perp}^{2}\left(q_{2 \perp}^{\prime} \times k_{2 \perp}\right)-k_{2 \perp}^{2}\left(q_{2 \perp}^{\prime} \times k_{3 \perp}\right)\right) \\
& +\frac{1}{2}\left(\left[\left(q_{3}^{+}-q_{2}^{+}\right) q_{1}^{\prime-}-q_{1}^{+} q_{3}^{\prime-}\right]\left(k_{1}^{2}-k_{2}^{2}\right)+\left(q_{3}^{+}-q_{2}^{+}\right) q_{2}^{\prime-} q_{2 \perp}^{\prime 2}\right)\left(q_{2 \perp} \times k_{3 \perp}\right)(. \mathrm{A}
\end{aligned}
$$

3) terms proportional to $\left(q_{1 \perp} \times q_{1 \perp}^{\prime}\right)\left(q_{3 \perp} \cdot q_{3 \perp}^{\prime}\right)$ are:

$$
\begin{aligned}
q_{2}^{+} q_{3}^{\prime-} & \left(q_{1}^{+} q_{1}^{\prime-}\left(q_{2 \perp} \times q_{2 \perp}^{\prime}\right)-q_{2 \perp} k_{2 \perp}\left(q_{2 \perp}^{\prime} \times k_{1 \perp}\right)+k_{1 \perp}^{2}\left(q_{2 \perp} \times k_{2 \perp}\right)-k_{2 \perp}^{2}\left(q_{2 \perp} \times k_{1 \perp}\right)\right) \\
& +\frac{1}{2}\left(\left[{q_{3}}^{+}\left({q_{1}^{\prime}}^{-}-{q_{2}^{\prime}}^{-}\right)-q_{1}^{+} q_{3}^{\prime-}\right]\left(k_{3 \perp}^{2}-k_{2 \perp}^{2}\right)+q_{2}^{+}\left({q_{1}^{\prime}}^{-}-q_{2}^{\prime-}\right) q_{2 \perp}^{2}\right)\left(q_{2 \perp}^{\prime} \times k_{1 \perp}\right)(\mathrm{A} .2
\end{aligned}
$$

4) terms proportional to $\left(q_{1 \perp} \cdot q_{1 \perp}^{\prime}\right)\left(q_{3 \perp} \cdot q_{3 \perp}^{\prime}\right)$ are:

$$
\begin{gathered}
\left(\frac{1}{2}\left(q_{3}^{+}{q_{1}^{\prime}}^{-}+q_{1}^{+} q_{3}^{\prime-}\right)\left(q_{1}^{+} q_{3}^{\prime-}+k_{2 \perp}^{2}\right)-q_{1}^{+} q_{2}^{+} q_{2}^{\prime-} q_{3}^{\prime-}\right) q_{2 \perp} q_{2 \perp}^{\prime} \\
-\frac{1}{2}\left(q_{3}^{+} q_{1}^{\prime-}+q_{1}^{+}{q_{3}^{\prime}}^{-}\right)\left(k_{1 \perp}^{2}-k_{2 \perp}^{2}\right)\left(k_{3 \perp}^{2}-k_{2 \perp}^{2}\right) \\
\quad-\frac{1}{2} q_{2}^{+} q_{1}^{\prime-} q_{2 \perp}^{2}\left(k_{1 \perp}^{2}-k_{2 \perp}^{2}\right) \\
-\frac{1}{2} q_{3}^{+} q_{2}^{\prime-} q_{2 \perp}^{\prime}\left(k_{3 \perp}^{2}-k_{2 \perp}^{2}\right) \\
-\frac{1}{2} q_{2}^{+} q_{2}^{\prime-} q_{2 \perp}^{2} q_{2 \perp}^{\prime}{ }^{2} .
\end{gathered}
$$

\section{A.5 Longitudinal integrals}

Let us now consider the longitudinal integrations over the variables $q_{1}^{\prime-}$ and $q_{3}^{\prime-}$, keeping $q_{1}{ }^{+}$and $q_{3}{ }^{+}$fixed. In the numerator we find, from (A.26) - (A.29), two groups of terms. The first one terms contains two powers, $q_{2}^{\prime-} q_{3}^{\prime-},{q_{1}^{\prime}}^{-}{q_{3}^{\prime-}}^{-}$, or $\left({q_{3}^{\prime-}}^{-2}\right.$, the second one single powers $q_{1}^{\prime-}, q_{2}^{\prime-}$, or $q_{3}^{\prime-}$. In (A.26) a third possible structure containing terms without any factors $q_{i}^{\prime-}$ could have appeared: but, as we have already mentioned, all contributions add up to zero. Combining with the prefactors in (A.1), all these terms have in the denominator (at least) two powers of $q_{1}^{\prime-}$ and $q_{3}^{\prime-}$, and thus lead to UV-convergent integrals. It is important to note that the only 'dangerous combination' $q_{1}^{\prime-} q_{2}^{\prime-}$ does not appear. This implies that the diagrams in Fig.5 alone are convergent, i.e. we do not need to sum over the permutations of the reggeized gluons.

Now let us take a closer look at the longitudinal integrals. Together with the denominators in (A.1) we are lead to compute the following integrals (using the principal value 
prescription, as explained in section 3.1) :

$$
\begin{aligned}
& I_{1}=\mathcal{P} \int d q_{1}^{\prime-} d q_{3}^{\prime-} \frac{1}{D_{1} D_{2} D_{3}} \frac{1}{q_{1}^{\prime-}} \\
& I_{2}=\mathcal{P} \int d q_{1}^{\prime-} d q_{3}^{\prime-} \frac{1}{D_{1} D_{2} D_{3}} \frac{1}{\left(q_{1}^{\prime}+q_{3}^{\prime}\right)^{-}} \\
& I_{3}=\mathcal{P} \int d q_{1}^{\prime-} d q_{3}^{\prime-} \frac{1}{D_{1} D_{2} D_{3}} \frac{q_{3}^{\prime-}}{q_{1}^{\prime-}\left(q_{1}^{\prime}+q_{3}^{\prime}\right)^{-}} \\
& I_{4}=\mathcal{P} \int d q_{1}^{\prime-} d q_{3}^{\prime-} \frac{1}{D_{1} D_{2} D_{3}} \frac{1}{\left(q_{1}^{\prime}+q_{3}^{\prime}\right)^{-} q_{3}^{\prime}} \\
& I_{5}=\mathcal{P} \int d q_{1}^{\prime-} d q_{3}^{\prime-} \frac{1}{D_{1} D_{2} D_{3}} \frac{1}{q_{1}^{\prime-} q_{3}^{\prime-}} \\
& I_{6}=\mathcal{P} \int d q_{1}^{\prime-} d q_{3}^{\prime-} \frac{1}{D_{1} D_{2} D_{3}} \frac{1}{q_{1}^{\prime-}\left(q_{1}^{\prime}+q_{3}^{\prime}\right)^{-}}
\end{aligned}
$$

with

$$
\begin{array}{lc}
D_{1}=k_{1}^{2}+i \epsilon= & q_{1}{ }^{+}\left(-q_{1}^{\prime}\right)^{-}+k_{1 \perp}^{2}+i \epsilon \\
D_{2}=k_{2}^{2}+i \epsilon= & q_{1}{ }^{+} q_{3}^{\prime}{ }^{-}+k_{2 \perp}^{2}+i \epsilon \\
D_{3}=k_{3}^{2}+i \epsilon= & q_{3}{ }^{+}\left(-q_{3}^{\prime}\right)^{-}+k_{3 \perp}^{2}+i \epsilon .
\end{array}
$$

In each case, we have to consider all possible signs of $q_{1}{ }^{+}$and $q_{3}{ }^{+}$. Let us denote the four regions $\left(q_{1}^{+}>0, q_{3}^{+}>0\right),\left(q_{1}^{+}<0, q_{3}^{+}<0\right),\left(q_{1}^{+}>0, q_{3}^{+}<0\right)$, and $\left(q_{1}^{+}<0, q_{3}^{+}>0\right)$ by $>>,<<,><,<>$, resp. As we have already said before, each $q_{1}^{\prime-}$-integral is ultraviolet convergent. The integrations are done by closing contours in the upper or lower half plane. We list the results:

$$
\begin{aligned}
& I_{1}=\mathcal{P} \int d q_{1}^{\prime-} d q_{3}^{\prime-} \frac{1}{D_{1} D_{2} D_{3}} \frac{1}{{q_{1}^{\prime}}^{-}}=\left\{\begin{array}{ll}
2(\pi i)^{2} \frac{1}{k_{1 \perp}^{2} D_{23}} & >>\text { or }<< \\
0 & ><\text { or }<>
\end{array},\right. \\
& I_{2}=\mathcal{P} \int d q_{1}^{\prime-} d q_{3}^{\prime-} \frac{1}{D_{1} D_{2} D_{3}} \frac{1}{\left(q_{1}^{\prime}+q_{3}^{\prime}\right)^{-}}=\left\{\begin{array}{ll}
2(\pi i)^{2} \frac{q_{3}^{+}}{D_{23} D_{13}} & >>\text { or }<< \\
0 & ><\text { or }<>
\end{array},\right.
\end{aligned}
$$

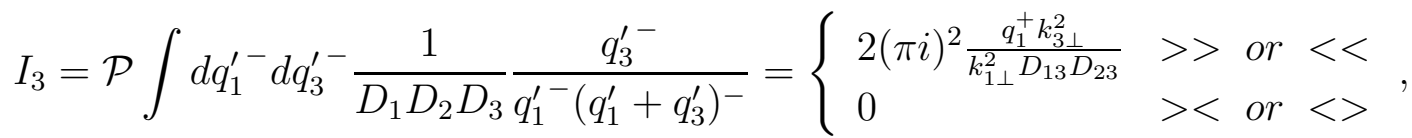

$$
\begin{aligned}
& I_{4}=\mathcal{P} \int d q_{1}^{\prime-} d q_{3}^{\prime-} \frac{1}{D_{1} D_{2} D_{3}} \frac{1}{\left(q_{1}^{\prime}+q_{3}^{\prime}\right)^{-} q_{3}^{\prime-}}=\left\{\begin{array}{ll}
2(\pi i)^{2} \frac{\left(q_{3}^{+}\right)^{2}}{k_{3 \perp}^{2} D_{23} D_{13}}-(\pi i)^{2} \frac{1}{k_{1 \perp}^{2} k_{2 \perp}^{2} k_{3 \perp}^{2}} & >>\text { or }<< \\
-(\pi i)^{2} \frac{1}{k_{1 \perp}^{2} k_{2 \perp}^{2} k_{3 \perp}^{2}} & ><\text { or }<>
\end{array} .\right. \\
& I_{5}=\mathcal{P} \int d q_{1}^{\prime-} d q_{3}^{\prime-} \frac{1}{D_{1} D_{2} D_{3}} \frac{1}{q_{1}^{\prime-} q_{3}^{\prime-}}=\left\{\begin{array}{ll}
2(\pi i)^{2} \frac{q_{3}^{+}}{k_{1 \perp}^{2} k_{3}^{2} D_{23}}-(\pi i)^{2} \frac{1}{k_{1 \perp}^{2} k_{2 \perp}^{2} k_{3 \perp}^{2}} & >>\text { or }<< \\
-(\pi i)^{2} \frac{k_{1}^{2}}{k_{1 \perp}^{2} k_{2 \perp}^{2} k_{3 \perp}^{2}} & ><\text { or }<>
\end{array},\right.
\end{aligned}
$$

and

$$
I_{6}=\mathcal{P} \int d q_{1}^{\prime-} d q_{3}^{\prime-} \frac{1}{D_{1} D_{2} D_{3}} \frac{1}{q_{1}^{\prime-}\left(q_{1}^{\prime}+q_{3}^{\prime}\right)^{-}}=\left\{\begin{array}{ll}
2(\pi i)^{2} \frac{q_{1}^{+} q_{3}^{+}}{k_{1 \perp}^{2} D_{13} D_{23}}-(\pi i)^{2} \frac{1}{k_{1 \perp}^{2} k_{2 \perp}^{2} k_{3 \perp}^{2}} & >>\text { or }<< \\
-(\pi i)^{2} \frac{1}{k_{1 \perp}^{2} k_{2 \perp}^{2} k_{3 \perp}^{2}} & ><\text { or }<>
\end{array} .\right.
$$


with

$$
D_{13}=q_{3}^{+} k_{1 \perp}^{2}+q_{1}^{+} k_{3 \perp}^{2}
$$

and

$$
D_{23}=q_{3}^{+} k_{2 \perp}^{2}+q_{1}^{+} k_{3 \perp}^{2} .
$$

In all cases, the upper lines on the rhs correspond to the case of equal sign: $q_{1}^{+}>0, q_{3}^{+}>0$ or $q_{1}^{+}<0, q_{3}^{+}<0$, the lower lines to opposite signs: $q_{1}^{+}>0, q_{3}^{+}<0$ or $q_{1}^{+}<0, q_{3}^{+}>0$.

Looking at these results we make a few important observations. First, one easily verifies that in all $I_{i}$, for the case of equal signs, the first term on the rhs could have been obtained by ignoring the poles from $1 /\left(q_{1}^{\prime}\right)^{-}, 1 /\left(q_{2}^{\prime}\right)^{-}$and $1 /\left(q_{3}^{\prime}\right)^{-}$, closing the contours of the $\left(q_{1}^{\prime}\right)^{-}$and $\left(q_{3}^{\prime}\right)^{-}$integrations in the upper half planes and picking the poles in $\left(q_{1}^{\prime}\right)^{-}$ and $\left(q_{3}^{\prime}\right)^{-}$arising from the denominators $D_{1}$ and $D_{3}$, resp., and finally multiplying by a factor $1 / 2$. Second, the integrals $I_{4}, I_{5}$, and $I_{6}$ contain, on the rhs, terms $\sim 1 / k_{1 \perp}^{2} k_{2 \perp}^{2} k_{3 \perp}^{2}$ which appear both for equal and mixed signs of the $q_{i}^{+}$. Combining with the $q_{i}^{+}$factors contained in the prefactors (47), their dependence upon $q_{i}^{+}$is always of the simple form $1 / q_{i}^{+} q_{j}^{+}(i \neq j)$ : when integrating over positive and negative values of $q_{i}^{+}$and $q_{j}^{+}$, these terms cancel. As result, we are left with only the first terms proportional to $1 / D_{23}$ or $1 / D_{13} D_{23}$, belonging to the equal sign regions. For the integrals $I_{2}, I_{3}, I_{4}$, and $I_{6}$ we encounter the denominators $D_{23}$ and $D_{13}$ : the first one results from inserting the $q_{3}^{\prime-}$ pole from $D_{3}$ into the gluon propagator $1 / D_{2}$, the second one from inserting the $q_{3}^{\prime-}$ pole (from $D_{3}$ ) and the $q_{1}^{\prime-}$ pole (from $\left.D_{1}\right)$ into the denominator $1 /\left(q_{1}^{\prime}+q_{3}\right)^{-}=-1 / q_{2}^{\prime-}$ (which had its origin in our use of the Ward identities).

We summarize these findings as follows:

(i) only the equal sign regions, $<<$ and $>>$, contribute,

(ii) apart from the overall factor $1 / 2$, the results are the same as obtained from taking residues of $D_{1}$ and $D_{3}$, i.e. by putting the lines $k_{1}$ and $k_{3}$ on-shell.

With these findings we can come back to the representation of the $3 \rightarrow 3$ kernel given by Fig. 4. Since the only diagram having simultaneously poles at $D_{1}=0$ and $D_{3}=0$ is the first diagram on the rhs of Fig. 4, only this diagram must be considered. We are thus left with the diagram illustrated in Fig.7 with the product $R P R \otimes R P P R \otimes R P R$, with the s-channel gluons $k_{1}$ and $k_{3}$ being on-shell.

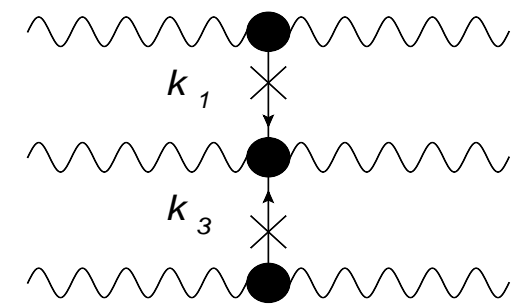

Fig.7: Final form of the $3 \rightarrow 3$ kernel: product of the effective RPR, RPPR, RPR vertices in the notation of Fig.3. The s-channel gluons with momenta $k_{1}$ and $k_{3}$ are on-shell.

Due to gauge invariance of the on-shell vertices we can use any gauge for the propagators of the gluons $k_{1}$ and $k_{3}$. 


\section{References}

[1] V.S. Fadin, E.A. Kuraev and L.N. Lipatov, Phys. Lett. B60 (1975) 50; E.A. Kuraev, L.N. Lipatov and V.S. Fadin, Zh. Eksp. Teor. Fiz. 71 (1976) 840 [Sov. Phys. JETP 44 (1976) 443]; 72 (1977) 377 [45 (1977) 199]; Ya.Ya. Balitskii and L.N. Lipatov, Sov. J. Nucl. Phys. 28 (1978) 822.

[2] V.S. Fadin, L.N. Lipatov, Phys. Lett. B429 (1998) 127;

M. Ciafaloni and G. Camici, Phys. Lett. B430 (1998) 349.

[3] A. V. Kotikov and L. N. Lipatov, Nucl. Phys. B 582 (2000) 19 hep-ph/0004008.

[4] V. S. Fadin, R. Fiore and A. Papa, Phys. Rev. D 60 (1999) 074025.

[5] V. S. Fadin and D. A. Gorbachev, Pis'ma v Zh. Eksp. Teor. Fiz. 71 (2000) 322 [JETP Letters 71 (2000) 222]; Phys. Atom. Nucl. 63 (2000) 2157 [Yad. Fiz. 63 (2000) 2253].

[6] V. S. Fadin and R. Fiore, Phys. Lett. B610 (2005) 61 [Erratum-ibid. B 621 (2005) 61]; Phys. Rev. D72 (2005) 014018.

[7] J. Bartels, L. N. Lipatov, A. Sabio Vera, Eur. Phys. J. C65 (2010) 587-605. arXiv:0807.0894 [hep-th]].

[8] J. Bartels, Nucl. Phys. B 175 (1980) 365;

J. Kwiecinski, M. Praszalowicz, Phys. Lett. B 94 (1980) 413.

[9] L. N. Lipatov High energy asymptotics of multi-colour QCD and exactly solvable lattice models, Padova preprint DFPD/93/TH/70, hep-th/9311037, unpublished; L. N. Lipatov, JETP Lett. 59 (1994) 596;

L. D. Faddeev, G. P. Korchemsky, Phys. Lett. B 342 (1995) 311

[10] L. N. Lipatov, J. Phys. A42 (2009) 304020. [arXiv:0902.1444 [hep-th]].

[11] J. Bartels, L. N. Lipatov, G. P. Vacca, Phys. Lett. B477 (2000) 178-186. hep-ph/9912423.

[12] V. S. Fadin, R. Fiore, Phys. Lett. B440 (1998) 359-366.

[13] L. N. Lipatov, Nucl. Phys. B 452 (1995) 369 [hep-ph/9502308];

L. N. Lipatov, Phys. Rept. 286 (1997) 131 [hep-ph/9610276].

[14] E. N. Antonov, L. N. Lipatov, E. A. Kuraev, I. O. Cherednikov, Nucl. Phys. B721 (2005) 111-135. hep-ph/0411185.

[15] J. Bartels, L. N. Lipatov and G. P. Vacca, arXiv:1205.2530 [hep-th].

[16] V. S. Fadin, R. Fiore, M. I. Kotsky, Phys. Lett. B387 (1996) 593; J. Blumlein, V. Ravindran and W.L. van Neerven, Phys. Rev. D58 (1998) 091502; V. Del Duca and E.W.N. Glover, JHEP 0110 (2001) 015.

[17] V. S. Fadin, R. Fiore, A. V. Grabovsky, A. Papa, Nucl. Phys. B784 (2007) 49. 
[18] V.S. Fadin, M.I. Kotsky and R. Fiore, Phys. Lett. B359 (1995) 181.

[19] R. E. Gerasimov, V. S. Fadin, Phys. Atom. Nucl. 73 (2010) 1214.

[20] V. N. Gribov, L. N. Lipatov and G. V. Frolov, Sov. J. Nucl. Phys. 12 (1971) 543 [Yad. Fiz. 12 (1970) 994].

[21] H. Cheng and T. T. Wu, Phys. Rev. D1 (1970) 2775.

[22] V. S. Fadin, R. Fiore and A. Papa, Phys. Lett. B 647 (2007) 179.

[23] J. Bartels, L. N. Lipatov and G. P. Vacca, Nucl. Phys. B 706 (2005) 391 hep-ph/0404110;

J. Bartels, L. N. Lipatov, M. Salvadore and G. P. Vacca, Nucl. Phys. B 726 (2005) 53 [hep-ph/0506235].

[24] V. S. Fadin, R. Fiore, A. V. Grabovsky and A. Papa, AIP Conf. Proc. 1350 (2011) 224.

[25] V. S. Fadin, M. G. Kozlov, A. V. Reznichenko, Phys. Atom. Nucl. 67 (2004) 359-375. hep-ph/0302224

[26] L.N. Lipatov and V.S. Fadin, Zh. Eksp. Teor. Fiz. Pisma 49 (1989) 311 [Sov. Phys. JETP Lett. 49 (1989) 352]; Yad. Fiz. 50, (1989) 1141 [Sov. J. Nucl. Phys. 50 (1989) $712]$.

[27] L. N. Lipatov, Nucl. Phys. Proc. Suppl. 79, 207 (1999). 